\title{
TWISTORS, KÄHLER MANIFOLDS, AND BIMEROMORPHIC GEOMETRY. I
}

\author{
CLAUDE LEBRUN
}

\section{INTRODUCTION}

The focus of this article concerns an interplay, known as the Penrose twistor correspondence $[2,25]$, between 4-dimensional conformal geometry and the theory of complex 3-folds. This correspondence will be seen to lead us naturally to new and sometimes surprising results on both sides of the balance sheet. On the Riemannian geometry side, we will prove new existence theorems for complete Kähler surfaces of scalar curvature zero by applying a relative form of KodairaSpencer deformation theory to the complex 3-fold associated with such a Kähler manifold of real dimension 4 . On the other hand, the correspondence will give us enough extra control on the structure of the complex 3-manifolds in question that, in an accompanying article [23] coauthored with Poon, we will be able to show that these small deformations of Moishezon manifolds are typically not bimeromorphic to any Kähler manifold, thereby providing counterexamples to a conjecture of Fujiki [8] to the effect that the class of complex manifolds that are bimeromorphic to Kähler manifolds is not stable under small deformations. ${ }^{1}$

The Penrose correspondence applies only to those Riemannian 4-manifolds that are half-conformally flat. An oriented Riemannian 4-manifold $(M, g)$ is said to be self-dual (respectively, anti-self-dual) if its Weyl conformal curvature $W$ satisfies $W=\star W$, (respectively, $W=-\star W$ ), where $W$ is considered as a bundle-valued 2 -form and $\star$ is the the Hodge star operator, and is called half-conformally-flat if it is either self-dual or anti-self-dual. ${ }^{2}$ This definition is conformally invariant: if $g$ is half-conformally-flat, so is $\alpha g$ for $\alpha$ any smooth positive function; therefore, it will sometimes be more appropriate to speak in terms of the conformal class $[g]:=\left\{\alpha g \mid \alpha: M \stackrel{C^{\infty}}{\rightarrow} \mathbb{R}^{+}\right\}$(also called the conformal metric) rather of the metric itself.

Received by the editors October 5, 1990.

1991 Mathematics Subject Classification. Primary 53C55; Secondary 32G04, 32J17, 32 L25.

Supported in part by NSF grant DMS-9003263.

${ }^{1}$ This result was simultaneously proved by F. Campana, using a slightly different class of deformations of the same spaces.

${ }^{2}$ Notice that changing the orientation of $M$ interchanges the notions of self-duality and antiself-duality. 
If $(M,[g])$ is a self-dual (respectively, anti-self-dual) conformal Riemannian 4-manifold, its twistor space is a complex 3-manifold $Z$ whose underlying smooth 6-manifold is the total space of the sphere bundle of the rank-three real vector bundle of anti-self-dual (respectively, self-dual) 2-forms:

$$
\begin{gathered}
S^{2} \rightarrow \quad \\
\qquad:=S\left(\wedge_{\mp}\right) \\
\downarrow \\
M
\end{gathered}
$$

(Recall that $\Lambda_{ \pm}$consists of those 2-forms $\omega$ satisfying $\star \omega= \pm \omega$.) We may then give $Z$ an almost complex structure $J: T Z \rightarrow T Z, J^{2}=-1$, by first observing that, for each $x \in M$, there is a natural one-to-one correspondence between $\wp^{-1}(x)$ and the set of g-orthogonal complex structures $\jmath: T_{x} M \rightarrow T_{x} M$ inducing the negative (respectively, the given) orientation on $T_{x} M$; namely, any such $J$ corresponds to the 2 -form $\omega_{j}$ defined by

$$
\omega_{\jmath}(\xi, \eta)=\frac{1}{\sqrt{2}} g(\xi, \jmath \eta) .
$$

Using the Levi-Civita connection of $g$, we may lift $\jmath$ to act on the horizontal subspace of $T Z$ at $\omega_{j}$; this defines a complex structure on the $g$-horizontal subbundle of $T Z$. Since the fibers of $\wp$ are, by construction, oriented metric 2spheres, we may extend this horizontal structure as an almost-complex structure on $Z$ by requiring that it preserve the vertical subspace and induce the usual structure there. Remarkably, this almost-complex structure is then conformally invariant and is integrable precisely because $(M, g)$ is, by assumption, halfconformally-flat.

Now a Kähler manifold of complex dimension 2 is anti-self-dual (with respect to the standard orientation) iff its scalar-curvature vanishes $[6,9]$. This should be considered rather remarkable insofar as neither the Kähler condition nor the scalar-curvature condition are themselves conformally invariant, and yet their coincidence is reflected by a conformally invariant property. The Penrose correspondence now provides an unexpected link between scalar-flat Kähler geometry in complex dimension 2 and the theory of complex 3-folds. As will be explained at length in $\S 5$, the speciality of the metric being scalar-flat Kähler is encoded by the twistor space $Z$ admitting half-anti-canonical divisors with certain properties [26]. It is this fact that will allow us to study scalar-flat Kähler geometry by Kodaira-Spencer theory.

Quite apart from the above discussion, there are a number of compelling reasons for the study of scalar-flat Kähler surfaces. For the physicist, ample motivation is provided by the observation [6] that such spaces are solutions of the Einstein-Maxwell equations

$$
R_{a b}=2 F_{a}^{c} F_{c b}-\frac{1}{2} F_{c d} F^{c d} g_{a b}
$$


governing the gravitational field in the presence of electromagnetism, the electromagnetic field being given here by $F=(\omega+P) / 2$, where $\omega$ and $P$ are the Kähler and Ricci forms, respectively. For the Riemannian geometer, an equally powerful source of motivation is provided by the fact that, on a compact 4manifold, any scalar-flat Kähler metric provides an absolute minimum of the $L^{2}$-norm of the curvature tensor as a functional the space of Riemannian metrics. And finally, for the complex geometer, the existence problem for Kähler metrics of constant scalar curvature on a given compact complex manifold is central to Calabi's critical metric program [4]. It is thus unsurprising that the classification of compact scalar-flat Kähler surfaces appears on Yau's famous problem list [30]. While direct attacks on the problem using methods of nonlinear elliptic PDE have yet to bear fruit, geometric methods related to the twistor approach developed herein have recently settled the problem for those compact manifolds admitting holomorphic vector fields [21, 22].

In this article, we will, by contrast, be interested in asymptotically flat scalarflat Kähler manifolds. By asymptotically flat, we mean here that our manifold $M$ is assumed to be noncompact and connected and that there is a compact set $K \subset M$ such that each component of $M-K$ is diffeomorphic to the complement of a ball in $\mathbb{R}^{4}$ in such a way that the metric $g$ on $M-K$ is of the form

$$
g=g_{\text {Euclidean }}+O\left(1 / r^{2}\right)
$$

in particular, the Kähler metric $g$ is assumed to be complete. The above hypothesis is strong enough to imply that the complex-structure tensor $J$ of $(M, g)$ satisfies $\nabla_{\text {Euclidean }} J=O\left(1 / r^{3}\right)$, so that, after an orthogonal transformation of $\mathbb{R}^{4}, J$ tends asymptotically to the standard complex structure on $\mathbb{C}^{2}=\mathbb{R}^{4}$. The complex surface may therefore be compactified by adding a rational curve of self-intersection 1 at each end, i.e., for each component of $M-K$. By surface classification, there is therefore only one end, and the compactified surface is $\mathbb{C P}_{2}$ blown up at a finite collection of points. The original noncompact complex surface $M$ is therefore $\mathbb{C}^{2}$ blown up at $n$ points, some of which may coincide.

Our problem thus reduces to that of constructing and classifying asymptotically Euclidean scalar-flat Kähler metrics on blowups of $\mathbb{C}^{2}$. For the case in which the $n$ given points are collinear, the present author has given explicit examples [20] of such metrics and their associated twistor spaces. ${ }^{3}$ In the present work, we will show that there is a metric of the desired type in each Kähler class provided that the given configuration of points is sufficiently close to a collinear configuration. Among metrics close to the explicit ones, we will also observe that the configuration and Kähler class determine the scalar-flat Kähler metric uniquely. In brief, this gives us a theorem of weak-Torelli type, stating

\footnotetext{
${ }^{3}$ By conformal compactification, these metrics also give explicit examples of self-dual metrics on the $\mathrm{n}$-fold connected sum $\mathbb{C P}_{2} \# \cdots \# \mathbb{C P}_{2}$ of complex projective planes. Note that abstract proofs of the existence of self-dual metrics on these spaces have previously been given by Floer [7] and Donaldson-Friedman [5].
} 
that the moduli space of our metrics can be locally identified with the space of configurations of $n$ points in $\mathbb{C}^{2}$ with positive weights, modulo similarity transformations. ${ }^{4}$ One might hope for a stronger existence and uniqueness result that would yield a "global Torelli theorem" for this problem.

\section{MODEL TWISTOR SPACES}

In this section, we will recall the explicit construction [20] of twistor spaces for self-dual metrics on $\mathbb{C P}_{2} \# \cdots \# \mathbb{C P}_{2}$ that admit a nontrivial Killing field. These examples are obtained as birational modifications of certain algebraic varieties, and therefore are, in particular, Moishezon spaces. They include the twistor spaces of the symmetric metrics on $S^{4}$ and $\mathbb{C P}_{2}$ [13] and of Poon's metrics on $2 \mathbb{C P}_{2}$ [27]. The construction was inspired by Hitchin's work on the twistor spaces of gravitational instantons [11].

Let $\rho: \mathbb{C P}_{1} \times \mathbb{C P}_{1} \rightarrow \mathbb{C P}_{1} \times \mathbb{C P}_{1}$ denote the antiholomorphic involution

$$
\left(\left[z_{0}, z_{1}\right],\left[\zeta_{0}, \zeta_{1}\right]\right) \mapsto\left(\left[\overline{\zeta_{0}}, \overline{\zeta_{1}}\right],\left[\overline{z_{0}}, \overline{z_{1}}\right]\right),
$$

and let $S \subset \mathbb{C P}_{1} \times \mathbb{C P}_{1}$ denote the fixed-point set of $\rho$. Then $[14,15]$ there is a twistorlike correspondence relating $\mathbb{C P}_{1} \times \mathbb{C P}_{1}-S$ and hyperbolic 3 -space; more precisely, the set of $\rho$-invariant, compact holomorphic curves in the generating homology class of $H_{2}\left(\mathbb{C P}_{1} \times \mathbb{C P}_{1}-S, \mathbb{Z}\right) \cong \mathbb{Z}$ gives a natural model for $\mathscr{H}^{3}$. Indeed, every holomorphic curve $C$ in $\mathbb{C P}_{1} \times \mathbb{C P}_{1}$ homologous to the diagonal $\mathbb{C P}_{1} \subset \mathbb{C P}_{1} \times \mathbb{C P}_{1}$ is the zero locus of some $P \in \Gamma\left(\mathbb{C P}_{1} \times\right.$ $\left.\mathbb{C P}_{1}, \mathscr{O}(1,1)\right) \cong \mathbb{C}^{4}$, uniquely determined up to a multiplicative constant; here $\mathscr{O}(k, \ell)$ denotes the holomorphic line bundle on $\mathbb{C P}_{1} \times \mathbb{C P}_{1}$ of Chern class $(k, \ell) \in H^{2}\left(\mathbb{C P}_{1} \times \mathbb{C P}_{1}, \mathbb{Z}\right) \cong \mathbb{Z} \oplus \mathbb{Z}$. The requirement that $C$ be $\rho$ invariant means that we may take $P$ to be real with respect to the induced conjugate-linear involution $\mathscr{O}(1,1) \rightarrow \mathscr{O}(1,1)$. Now, up to a constant factor, $\Gamma\left(\mathbb{C P}_{1} \times \mathbb{C P}_{1}, \mathscr{O}(1,1)\right) \cong \mathbb{C}^{4}$ carries a natural nondegenerate quadratic form, specified by the requirement that the image of $\mathbb{C P}_{1} \times \mathbb{C P}_{1}$ under the Kodaira embedding map

$$
\mathbb{C P}_{1} \times \mathbb{C P}_{1} \rightarrow \mathbb{P}\left[\Gamma\left(\mathbb{C P}_{1} \times \mathbb{C P}_{1}, \mathscr{O}(1,1)\right)\right]^{*}
$$

be its set of null projective covectors; the corresponding real quadratic form on the real subspace $\Gamma\left(\mathbb{C P}_{1} \times \mathbb{C P}_{1}, \mathscr{O}(1,1)\right)_{\rho} \cong \mathbb{R}^{4}$ thus has the 2-sphere $S$ as its projective null cone, and so (for topological reasons) must be of Minkowski signature (+- - ). A $\rho$-invariant section $P$ of $\mathscr{O}(1,1)$ now defines a curve $C$ disjoint from $S$ iff it has positive Minkowski squarenorm. As such a section may always be multiplied by a nonzero constant without altering the corresponding curve $C \subset \mathbb{C P}_{1} \times \mathbb{C P}_{1}$, we conclude that the set of $\rho$-invariant, compact holomorphic curves in the generating homology class $1 \in$ $H_{2}\left(\mathbb{C P}_{1} \times \mathbb{C P}_{1}-S, \mathbb{Z}\right) \cong \mathbb{Z}$ are faithfully parameterized by future-pointing

\footnotetext{
${ }^{4}$ The weights, which represent the areas of the corresponding exceptional divisors, are unchanged by any unitary isometry of $\mathbb{C}^{2}$ and rescale by $\lambda^{2}$ under the homothety $\vec{z} \mapsto \lambda \vec{z}, \lambda \in \mathbb{R}^{+}$.
} 
timelike unit vectors in Minkowski space. But the latter hyperboloid is, of course, isometric to $\mathscr{H}^{3}$.

All of the hyperbolic geometry of $\mathscr{H}^{3}$ is completely encoded by this "minitwistor" model. For example, a hyperbolic geodesic, being a 2-plane section of the above hyperboloid, is exactly the set of curves through a $\rho$-conjugate pair of points in $\mathbb{C P}_{1} \times \mathbb{C P}_{1}-S$, namely, those hyperplane sections of $\mathbb{C P}_{1} \times \mathbb{C P}_{1} \subset$ $\mathbb{C P}_{3}$ for which the hyperplane contains the fixed real 2-plane. Concretely, the resulting pair of points $\{[\zeta],[z]),([\bar{z}],[\bar{\zeta}]\} \subset \mathbb{C P}_{1} \times \mathbb{C P}_{1}$ is given by the two end points $[\zeta],[\bar{z}] \in S^{2}$ of the geodesic at hyperbolic infinity $S$, which we identify with $\mathbb{C P}_{1}$ in the obvious manner. We can therefore identify $\mathbb{C P}_{1} \times$ $\mathbb{C P}_{1}-S$ with the space of oriented geodesics in $\mathscr{H}^{3}$ by thinking of the first and second factors as respectively representing the initial and final end points at infinity of hyperbolic geodesics. This has the following consequence, which has a critical rôle in the discussion that follows: if $C_{1}$ and $C_{2}$ are any two $\rho$ invariant curves in $\mathbb{C P}_{1} \times \mathbb{C P}_{1}-S$ that lie in the discussed homology class, then their two intersection points may be systematically labeled so that one "points from $C_{1}$ to $C_{2}$," while the other "points from $C_{2}$ to $C_{1}$."

Let $p_{1}, \ldots, p_{n}$ be arbitrary, distinct points in $\mathscr{H}^{3}$ that are in general position in the sense that that no three of these $n$ points are on a common hyperbolic geodesic. Let $P_{1}, \ldots, P_{n} \in \Gamma\left(\mathbb{C P}_{1} \times \mathbb{C P}_{1}, \mathscr{O}(1,1)\right)$ be the corresponding polynomials, and let $C_{1}, \ldots, C_{n} \subset \mathbb{C P}_{1} \times \mathbb{C P}_{1}-S$ be the $n$ curves they define. Let $\mathscr{B}$ denote the total space of the $\mathbb{C P}_{2}$-bundle

$$
\mathscr{B}:=\mathbb{P}(\mathscr{O}(n-1,1) \oplus \mathscr{O}(1, n-1) \oplus \mathscr{O}) \stackrel{\pi}{\longrightarrow} \mathbb{C P}_{1} \times \mathbb{C P}_{1}
$$

and define an algebraic variety $\tilde{Z} \subset \mathscr{B}$ by the equation $x y=t^{2} \prod_{j=1}^{n} P_{j}$, where $x \in \mathscr{O}(n-1,1), y \in \mathscr{O}(1, n-1)$, and $t \in \mathscr{O}:=\mathscr{O}(0,0)$. Our assumption on the $p_{1}, \ldots, p_{n}$ exactly amounts to requiring that no three of the curves defined by the $P_{j}$ have a common intersection, so that the locus in $\mathbb{C P} \mathbb{P}_{1} \times \mathbb{C P}_{1}$ defined by $\Pi P_{j}$ therefore has only normal crossing singularities. These intersection points in $\{x=y=0\} \subset \mathscr{B}$ may be checked to be the the only singular points of the hypersurface $\widetilde{Z}$.

There is a canonical antiholomorphic identification of $\rho^{*} \mathscr{O}(k, \ell)$ with $\mathscr{O}(\ell, k)$, and this induces an antiholomorphic involution of $\mathscr{B}$, and hence of $\widetilde{Z}$. Let us denote the latter involution by $\widetilde{\sigma}: \widetilde{Z} \rightarrow \widetilde{Z}$.

We will now construct a complex 3 -manifold $Z$ bimeromorphic to $\tilde{Z}$. First of all, notice that the two surfaces $x=t=0$ and $y=t=0$ are contained in $\widetilde{Z}$, and that, identifying them with the base $\mathbb{C P}_{1} \times \mathbb{C P}_{1}$ via the canonical projection, their normal bundles relative to $\widetilde{Z}$ are $\mathscr{O}(-1,1-n)$ and $\mathscr{O}(1-n,-1)$, respectively. Each, therefore, can be blown down to yield a rational curve $\mathbb{C P}_{1}$, both of which will have normal bundle $\mathscr{O}(1-n) \oplus \mathscr{O}(1-n)$. These rational curves will be called $\mathscr{C}$ and $\overline{\mathscr{C}}$, respectively. This blowing down constitutes the first step in the construction of $Z$ from $\widetilde{Z}$. 
We complete the construction by making "small resolutions" of the the singular points of $\widetilde{Z}$. To do this, notice that the singular points of $\widetilde{Z}$ occur in pairs, which are "conjugate" via $\rho$. Each such singularity may be put in the canonical form $x y=z w$ by choosing local coordinates $z$ and $w$ on $\mathbb{C P}_{1} \times \mathbb{C P}_{1}$ centered at the given crossing point of $C_{j}$ and $C_{k}$, and a "small resolution" of this singularity, therefore, can be obtained by using the map

$$
\begin{aligned}
\mathscr{O}(-1) \oplus \mathscr{O}(-1) & \rightarrow \mathbb{C}^{4} \\
\left.(u, v)\right|_{\left[\zeta^{0}, \zeta^{1}\right]} & \mapsto(x, y, z, w):=\left(u \zeta^{0}, v \zeta^{1}, u \zeta^{1}, v \zeta^{0}\right)
\end{aligned}
$$

as a local model; here $\mathscr{O}(-1)$ denotes the "tautological" line bundle over $\mathbb{C P}_{1}$. (The singular point is thus replaced with a rational curve $\mathbb{C P}_{1}$.) Inspection of this resolution reveals that it just amounts to blowing up $\mathscr{B}$ at a quadratic singularity of the hypersurface $\widetilde{Z}$, taking the proper transform of $\widetilde{Z}$, and then collapsing a chosen factor of the resulting exceptional 2-quadric $\mathbb{Q}_{2} \cong \mathbb{C P}_{1} \times \mathbb{C P}_{1}$ so as to produce a rational curve. As this depends on the choice of a factor of $\mathbb{C P}_{1} \times \mathbb{C P}_{1}$ at each singularity, we will insist on the following rule: at the intersection point $q_{j k}$ of a pair of real curves $C_{j}$ and $C_{k}$ that "points from $C_{j}$ to $C_{k}$," the resolutions should be obtained from the above prescription by letting $z$ define $C_{j}$ and letting $w$ define $C_{k}$, while $x$ and $y$ are again coordinates on $\mathscr{O}(n-1,1)$ and $\mathscr{O}(1, n-1)$, respectively. With this choice of conventions, the involution $\tilde{\sigma}$ induces an involution $\sigma$ of the constructed complex 3-fold $Z$.

Let us now draw a more explicit road map of the route between $Z$ and $\tilde{Z}$. To do this, we let $\widehat{\mathscr{B}}$ denote the blow-up of $\mathscr{B}$ at the $n(n-1)$ singular points of $\widetilde{Z}$, and let $b: \widehat{\mathscr{B}} \rightarrow \mathscr{B}$ denote the associated blowing-down map. Let $\widehat{Z} \subset \widehat{\mathscr{B}}$ denote the proper transform of $\widetilde{Z}$. Then $\widehat{Z}$ is obtained from $Z$ by blowing up $n(n-1)+2$ rational curves; we let $a: \widehat{Z} \rightarrow Z$ denote the associated blowing-down map. The diagram

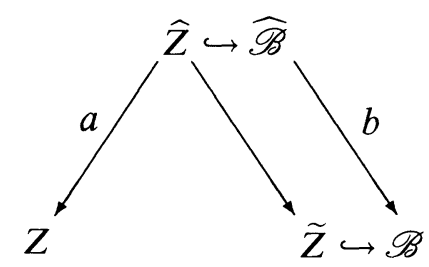

now provides the promised road map.

\section{3. Сономоlogy computations}

In this section, we compute the cohomology of the the tangent sheaf of any twistor space $Z$ arising by the construction of the previous section. More importantly, we will also be able to compute the cohomology of certain relative tangent sheaves that will feature prominently in the deformation arguments of $\S 4$. 
Proposition 1. Let $n \geq 3$. Then

$$
h^{j}(\widehat{Z}, \mathscr{O}(T \widehat{Z}))=\left\{\begin{array}{cl}
1 & j=0 \\
3 n-6 & j=1 \\
0 & j \geq 2
\end{array}\right.
$$

Proof. Our approach is based on exploiting the short exact sequence

$$
0 \rightarrow \mathscr{O}(T \widehat{Z}) \rightarrow \mathscr{O}(T \widehat{\mathscr{B}}) \rightarrow \mathscr{O}(\nu) \rightarrow 0
$$

of sheaves on $\widehat{Z}$, where $\nu$ is the normal bundle of $\widehat{Z} \subset \widehat{\mathscr{B}}$.

Let $V \rightarrow \mathbb{C P}_{1} \times \mathbb{C P}_{1}$ denote the vector bundle

$$
V:=\mathscr{O}(n-1,1) \oplus \mathscr{O}(1, n-1) \oplus \mathscr{O},
$$

and let $\mathscr{O}(0,0,-1)$ denote the tautological line bundle over $\mathscr{B}$, corresponding to the principal $\mathbb{C}_{*}$-bundle $V-0_{\mathbb{C P}_{1} \times \mathbb{C P}_{1}} \rightarrow \mathbb{P}(V)$. For any $j, k, \ell \in \mathbb{Z}$, we set

$$
\mathscr{O}(j, k, \ell):=[\mathscr{O}(0,0,-1)]^{* \otimes \ell} \otimes \pi^{*} \mathscr{O}(j, k) \text {. }
$$

Let $\mathscr{I} \subset \mathscr{O}_{\widehat{\mathscr{B}}}$ denote the ideal sheaf of $\widehat{Z} \subset \widehat{\mathscr{B}}$, and let $\mathscr{J} \subset \mathscr{O}_{\mathscr{B}}$ denote the ideal sheaf of $\widetilde{Z} \subset \mathscr{B}$. Let $\mathscr{K} \subset \mathscr{O}_{\mathscr{B}}$ denote the ideal sheaf of the set $\mathscr{Q}:=\left\{q_{1 \mid 1}, \ldots, q_{n \mid(n-1)}\right\}=\bigcup_{j \neq k}\left[C_{j} \cap C_{k}\right]$ of points blown up by $b$, and let $E$ denote the divisor line bundle of the exceptional set $b^{-1}[\mathscr{Q}] \subset \widehat{\mathscr{B}}$. Since $\mathscr{J} \cong \mathscr{O}(-n,-n,-2)$ and the singularities of $\widetilde{Z}$ are all quadratic, we have

$$
\mathscr{I}=E^{2} \otimes b^{*} \mathscr{J} \cong E^{2} \otimes b^{*} \mathscr{O}(-n,-n,-2) \text {. }
$$

Again, let $\nu$ denote the normal bundle of $\widehat{Z} \subset \widehat{\mathscr{B}}$. We then have an exact sequence

$$
0 \rightarrow \mathscr{O} \rightarrow \mathscr{H} a m(\mathscr{I}, \mathscr{O}) \rightarrow \mathscr{O}_{\widehat{Z}}(\nu) \rightarrow 0
$$

of sheaves on $\widehat{B}$, which we can rewrite as

$$
0 \rightarrow \mathscr{O} \rightarrow E^{-2} \otimes b^{*} \mathscr{O}(n, n, 2) \rightarrow \mathscr{O}_{\widehat{Z}}(\nu) \rightarrow 0
$$

in light of the above remarks. However, the direct images of $\mathscr{O}$ and $E^{-2} \otimes$ $b^{*} \mathscr{O}(n, n, 2)$ via $b$ are respectively given by

$$
b_{*}^{j} \mathscr{O}= \begin{cases}\mathscr{O} & j=0 \\ 0 & j \neq 0\end{cases}
$$

and

$$
b_{*}^{j}\left[E^{-2} \otimes b^{*} \mathscr{O}(n, n, 2)\right]= \begin{cases}\mathscr{K}^{2} \otimes \mathscr{O}(n, n, 2) & j=0, \\ 0 & j \neq 0 .\end{cases}
$$

But the direct images of $\mathscr{O}$ via $\pi: \mathscr{B} \rightarrow \mathbb{C P}_{1} \times \mathbb{C P}_{1}$ are given by

$$
\pi_{*}^{j} \mathscr{O}= \begin{cases}\mathscr{O} & j=0, \\ 0 & j \neq 0,\end{cases}
$$


so that

$$
H^{j}(\mathscr{B}, \mathscr{O})=H^{j}\left(\mathbb{C P}_{1} \times \mathbb{C P}_{1}, \mathscr{O}\right)= \begin{cases}\mathbb{C} & j=0, \\ 0 & j \neq 0\end{cases}
$$

by the Leray spectral sequence of $\pi$. Thus, using the Leray spectral sequence of $b$ and the long exact sequence of (2), we have

$$
H^{j}(\widehat{Z}, \nu)= \begin{cases}H^{0}\left(\mathscr{B}, \mathscr{K}^{2} \otimes \mathscr{O}(n, n, 2)\right) / \mathbb{C} & j=0, \\ H^{j}\left(\mathscr{B}, \mathscr{K}^{2} \otimes \mathscr{O}(n, n, 2)\right) & j \neq 0 .\end{cases}
$$

On the other hand, the direct images of $\mathscr{O}(T \widehat{\mathscr{B}})$ via $b$ are given by

$$
b_{*}^{j} \mathscr{O}(T \widehat{\mathscr{B}})= \begin{cases}\mathscr{K}(T \mathscr{B}) & j=0, \\ 0 & j \neq 0,\end{cases}
$$

whereas those of $\mathscr{O}(T \widehat{\mathscr{B}}) \otimes \mathscr{I}=\mathscr{O}(T \widehat{\mathscr{B}}) \otimes E^{2} \otimes b^{*} \mathscr{J}$ are given by

$$
b_{*}^{j} \mathscr{J}(T \widehat{\mathscr{B}})= \begin{cases}\mathscr{J}(T \mathscr{B}) & j=0, \\ 0 & j \neq 0,\end{cases}
$$

Now $T \mathscr{B}$ fits into the exact sequence

$$
0 \rightarrow T_{\pi} \mathscr{B} \rightarrow T \mathscr{B} \rightarrow \pi^{*}[\mathscr{O}(2,0) \oplus \mathscr{O}(0,2)] \rightarrow 0,
$$

where the vertical tangent bundle $T_{\pi} \mathscr{B}$ in turn fits into the exact sequence

$$
0 \rightarrow \mathscr{O} \rightarrow V \otimes \mathscr{O}(0,0,1) \rightarrow T_{\pi} \mathscr{B} \rightarrow 0
$$

Thus the direct images via $\pi$ of $\mathscr{J}(T \mathscr{B})=\mathscr{O}(T \mathscr{B}) \otimes \mathscr{O}(-n,-n,-2)$ all vanish, since $H^{j}\left(\mathbb{C P}_{2}, \mathscr{O}(-1)\right)=H^{j}\left(\mathbb{C P}_{2}, \mathscr{O}(-2)\right)=0$

$$
H^{j}(\widehat{\mathscr{B}}, \mathscr{I}(T \widehat{\mathscr{B}}))=H^{j}(\mathscr{B}, \mathscr{J}(T \mathscr{B}))=0 \quad \forall j \in \mathbb{Z}
$$

and the exact sequence

$$
0 \rightarrow \mathscr{I}(T \widehat{\mathscr{B}}) \rightarrow \mathscr{O}(T \widehat{\mathscr{B}}) \rightarrow \mathscr{O}_{\widehat{Z}}(T \widehat{\mathscr{B}}) \rightarrow 0
$$

of sheaves on $\mathscr{B}$, together with the Leray spectral sequence of $b$ therefore yields

$$
H^{j}(\widehat{Z}, T \widehat{\mathscr{B}})=H^{j}(\mathscr{B}, \mathscr{K}(T \mathscr{B}))
$$

We now need to compute $H^{j}\left(\mathscr{B}, \mathscr{K}^{2} \otimes \mathscr{O}(n, n, 2)\right)$ and $H^{j}(\mathscr{B}, \mathscr{K}(T \mathscr{B}))$. We do this by exploiting the short exact sequences

$$
\left.0 \rightarrow \mathscr{K}^{2} \otimes \mathscr{O}(n, n, 2) \rightarrow \mathscr{O}(n, n, 2) \rightarrow J^{1} \mathscr{O}(n, n, 2)\right|_{\mathscr{Q}} \rightarrow 0
$$

and

$$
\left.0 \rightarrow \mathscr{K}(T \mathscr{B}) \rightarrow \mathscr{O}(T \mathscr{B}) \rightarrow T \mathscr{B}\right|_{\mathscr{Q}} \rightarrow 0
$$


The cohomology groups $H^{j}(\mathscr{B}, \mathscr{O}(n, n, 2))$ are easily read from the Leray spectral sequence of $\pi$. Indeed,

$$
\pi_{*}^{j} \mathscr{O}(n, n, 2)= \begin{cases}\mathscr{O}(n, n) \otimes \bigodot^{2} V^{*} & j=0, \\ 0 & j \neq 0,\end{cases}
$$

so that

$$
H^{j}(\mathscr{B}, \mathscr{O}(n, n, 2))=H^{j}\left(\mathbb{C P}_{1} \times \mathbb{C P}_{1}, \mathscr{O}(n, n) \otimes \bigodot^{2} V^{*}\right)
$$

But since

$$
\begin{aligned}
\bigodot^{2} V^{*}= & \mathscr{O} \oplus \mathscr{O}(1-n,-1) \oplus \mathscr{O}(-1,1-n) \\
& \oplus \mathscr{O}(2-2 n,-2) \oplus \mathscr{O}(-2,2-2 n) \oplus \mathscr{O}(-n,-n),
\end{aligned}
$$

we read that

$$
\begin{aligned}
H^{0}(\mathscr{B}, \mathscr{O}(n, n, 2)) & =H^{0}\left(\mathbb{C P}_{1} \times \mathbb{C P}_{1}, \mathscr{O}(n, n) \oplus \mathscr{O}(1, n-1)\right. \\
& \cong \mathbb{C}^{n^{2}+6 n+2}
\end{aligned}
$$

and

$$
\begin{aligned}
H^{1}(\mathscr{B}, \mathscr{O}(n, n, 2)) & =H^{1}\left(\mathbb{C P}_{1} \times \mathbb{C P}_{1}, \mathscr{O}(2-n, n-2) \oplus \mathscr{O}(n-2,2-n)\right) \\
& \cong \begin{cases}0 & n<4, \\
\mathbb{C}^{2(n-1)(n-3)} & n \geq 4,\end{cases}
\end{aligned}
$$

whereas $H^{j}(\mathscr{B}, \mathscr{O}(n, n, 2))=0 \forall j>1$. As a consequence, one sees, in particular, that

$$
H^{j}(\widehat{Z}, \mathscr{O}(\nu))=0 \quad \forall j>1 .
$$

On the other hand, the exact sequences (3) and (4) allow us to compute the cohomology groups $H^{j}(\mathscr{B}, \mathscr{O}(T \mathscr{B}))$. Indeed, note that

$$
\begin{aligned}
H^{j}\left(\mathscr{B}, \mathscr{O}\left(T_{\pi} \mathscr{B}\right)\right) \\
\quad=H^{j}\left(\mathbb{C P}_{1} \times \mathbb{C P}_{1}, \mathscr{E} n d_{0}(V)\right) \\
\quad=H^{j}\left(\bigoplus\left\{\begin{array}{ccc}
\mathscr{O} & \mathscr{O}(n-1,1) & \mathscr{O}(1, n-1) \\
\mathscr{O}(1-n,-1) & \mathscr{O} & \mathscr{O}(2-n, n-2) \\
\mathscr{O}(-1,1-n) & \mathscr{O}(n-2,2-n) & 0
\end{array}\right\}\right),
\end{aligned}
$$

where $\operatorname{End}_{0}(V)$ is the sheaf of tracefree endomorphisms of $V$, whereas

$$
H^{j}\left(\mathscr{B}, \pi^{*}(\mathscr{O}(2,0) \oplus \mathscr{O}(0,2))\right) \cong \begin{cases}\mathbb{C}^{6} & j=0, \\ 0 & j \neq 0 .\end{cases}
$$

Thus

$$
H^{0}(\mathscr{B}, \mathscr{O}(T \mathscr{B})) \cong \mathbb{C}^{4 n+8},
$$




$$
\begin{aligned}
H^{1}(\mathscr{B}, \mathscr{O}(T \mathscr{B})) & =H^{1}\left(\mathbb{C P}_{1} \times \mathbb{C P}_{1}, \mathscr{O}(2-n, n-2) \oplus \mathscr{O}(n-2,2-n)\right) \\
& \cong \begin{cases}0 & n<4, \\
\mathbb{C}^{2(n-1)(n-3)} & n \geq 4,\end{cases}
\end{aligned}
$$

and

$$
H^{j}(\mathscr{B}, \mathscr{O}(T \mathscr{B}))=0 \quad \forall j>1 .
$$

From (5) and (7), we now get

$$
H^{j}(\widehat{Z}, \mathscr{O}(T \widehat{\mathscr{B}}))=0 \quad \forall j>1 .
$$

To finish the proof, we must explicitly understand how the natural map $H^{1}(\widehat{Z}, \mathscr{O}(T \widehat{\mathscr{B}})) \rightarrow H^{1}(\widehat{Z}, \mathscr{O}(\nu))$ fit in with the above exact sequences. We have been thinking of the normal bundle $\nu$ as the restriction of the divisor line bundle of $\widehat{Z} \subset \mathscr{B}$ to $\widehat{Z}$. In these terms, then, the map $\mathscr{O}(T \widehat{\mathscr{B}}) \rightarrow \mathscr{O}(\nu)$ may be thought of as sending a vector field to the corresponding directional derivative of the canonical section of the divisor line bundle, evaluated along $\widehat{Z}$. But, taking direct images via $b$, the canonical section of the divisor corresponds to a section of $\mathscr{O}(n, n, 2)$ that vanishes at $\widetilde{Z}$ and to first order at $\mathscr{Q}$, namely, $f=x y-t^{2} P_{1} \cdots P_{n}$. Interpreting $d f$ as a 1 -form on the total space of $\mathscr{O}(-1,-1,-1)$, the long exact sequence of $(1)$ therefore becomes

$$
\begin{aligned}
0 & \rightarrow H^{0}(\widehat{Z}, \mathscr{O}(T \widehat{Z})) \rightarrow H^{0}(\mathscr{B}, \mathscr{K}(T \mathscr{B})) \\
& \stackrel{d f}{\rightarrow} H^{0}\left(\mathscr{B}, \mathscr{K}^{2} \otimes \mathscr{O}(n, n, 2)\right) / \mathbb{C} \rightarrow \cdots \rightarrow H^{j}(\widehat{Z}, \mathscr{O}(T \widehat{Z})) \\
& \rightarrow H^{j}(\mathscr{B}, \mathscr{K}(T \mathscr{B})) \stackrel{d f}{\rightarrow} H^{j}\left(\mathscr{B}, \mathscr{K}^{2} \otimes \mathscr{O}(n, n, 2)\right) \rightarrow \cdots .
\end{aligned}
$$

We will now calculate $H^{0}(\mathscr{B}, \mathscr{K}(T \mathscr{B}))$ and $H^{0}\left(\mathscr{B}, \mathscr{K}^{2} \otimes \mathscr{O}(n, n, 2)\right)$ and show that both are two-dimensional.

Indeed, suppose that $\xi$ is a holomorphic vector field on $\mathscr{B}$ that vanishes at $\mathscr{Q} \subset \mathbb{C P}_{1} \times \mathbb{C P}_{1} \subset \mathscr{B}$, where $\mathbb{C P}_{1} \times \mathbb{C P}_{1}$ represents the locus $x=y=$ 0 . Its normal and tangential components along $\mathbb{C P}_{1} \times \mathbb{C P}_{1}$, respectively, are sections of $\mathscr{O}(n-1,1) \oplus \mathscr{O}(1, n-1)$ and $\mathscr{O}(2,0) \oplus \mathscr{O}(0,2)$ vanishing at each intersection $C_{j} \cap C_{k}, j \neq k$, where, as before, $C_{j} \subset \mathbb{C P}_{1} \times \mathbb{C P}_{1}$ is given by $P_{j}=0$. Since the $C_{j} \cong \mathbb{C P}_{1}$ represents the class $(1,1) \in H^{2}\left(\mathbb{C P}_{1} \times \mathbb{C P}_{1}, \mathbb{Z}\right) \cong$ $\mathbb{Z} \oplus \mathbb{Z}$, and since the intersections of $C_{j}$ with the other curves $C_{k}$ give us $2 n-2$ distinct points by the general position assumption regarding the $p_{j} \in \mathscr{H}^{3}$ made in $\S 2$, it follows that the vector field $\xi$ evaluated along $C_{j}$ has, as its component normal to $\mathbb{C P}_{1} \times \mathbb{C P}_{1} \subset \mathscr{B}$, a section of $\mathscr{O}(n) \oplus \mathscr{O}(n)$ vanishing at $2 n-2$ points, and so, since $n>2$ by assumption, this normal component vanishes identically on $\bigcup_{j=1}^{n} C_{j}$; hence the component normal to $\mathbb{C P}_{1} \times \mathbb{C P}_{1}$ is a section of $[\mathscr{O}(n-1,1) \oplus \mathscr{O}(1, n-1)] \otimes \mathscr{O}(-n,-n)=\mathscr{O}(-1,1-n) \oplus \mathscr{O}(1-n,-1)$, and so must vanish. Similarly, the component of $\xi$ tangent to $\mathbb{C P}_{1} \times \mathbb{C P}_{1}$ restricts to $C_{j}$ as a section of $[\mathscr{O}(2) \oplus \mathscr{O}(2)]$ with $2 n-2$ zeroes, and so vanishes on $\cup C_{j}$; on $\mathbb{C P}_{1} \times \mathbb{C P}_{1}$, it is therefore a section of $[\mathscr{O}(2,0) \oplus \mathscr{O}(0,2)] \otimes$ 
$\mathscr{O}(-n,-n)$, and thus vanishes identically on $\mathbb{C P}_{1} \times \mathbb{C P}_{1}$. By $(10)$, this means that $\xi$ is a linear combination of $x \frac{\partial}{\partial x}$ and $y \frac{\partial}{\partial y}$, so that

$$
h^{0}(\mathscr{B}, \mathscr{K}(T \mathscr{B}))=2 \text {, }
$$

as claimed.

In the same spirit, let $\varphi$ be a section of $\mathscr{O}(n, n, 2)$ over $\mathscr{B}$ such that $\varphi$ and $d \varphi$ vanish at all the points of $\mathscr{Q}=\bigcup_{j \neq k}\left[C_{j} \cap C_{k}\right]$. The restriction of $\varphi$ to $C_{j}$ is then a section of $\mathscr{O}(2 n)$ vanishing along with its derivative at $2 n-2$ points, and, since $n>2$, therefore vanishes identically on each $C_{j}$. The restriction of $\varphi$ to $\mathbb{C P}_{1} \times \mathbb{C P}_{1}$, therefore, is a section of $\mathscr{O}(n, n) \otimes \mathscr{O}(-n,-n)=\mathscr{O}$, showing that $\left.\varphi\right|_{\mathbb{C P}_{1} \times \mathbb{C P}_{1}}$ is of the form $c P_{1} \cdots P_{n}$ for some $c \in \mathbb{C}$. The normal derivative of $\varphi-c P_{1} \cdots P_{n}$ along $\mathbb{C P}_{1} \times \mathbb{C P}_{1}$ is now a section of $\mathscr{O}(n, n) \otimes$ $[\mathscr{O}(1-n,-1) \oplus \mathscr{O}(-1,1-n)]=\mathscr{O}(n-1,1) \oplus \mathscr{O}(1-n, 1)$ that vanishes at $\mathscr{Q}$. By the same argument as before, this normal derivative then vanishes on all of $\mathbb{C P}_{1} \times \mathbb{C P}_{1}$. By (8), we therefore conclude that $\varphi$ is a linear combination of $t^{2} P_{1} \cdots P_{n}$ and $x y$, so that

$$
h^{0}\left(\mathscr{B}, \mathscr{K}^{2} \otimes \mathscr{O}(n, n, 2)\right)=2,
$$

as claimed.

Now the restriction of $d f$ to the fibers of $\pi$ gives us a well-defined section $d_{\pi} f \in \Gamma\left(\mathbb{C P}_{1} \times \mathbb{C P}_{1}, \bigodot^{2} V^{*} \otimes \mathscr{O}(n, n)\right)$, and, by inspection of the above bases for $H^{0}(\mathscr{B}, \mathscr{H}(T \mathscr{B}))$ and $H^{0}\left(\mathscr{B}, \mathscr{K}^{2} \otimes \mathscr{O}(n, n, 2)\right)$, we observe that

$$
d_{\pi} f=x d y+y d x: H^{0}(\mathscr{B}, \mathscr{K}(T \mathscr{B})) \rightarrow H^{0}\left(\mathscr{B}, \mathscr{K}^{2} \otimes \mathscr{O}(n, n, 2)\right)
$$

is an isomorphism. It follows from the exact sequence (12) that

$$
h^{0}(\widehat{Z}, \mathscr{O}(T \widehat{Z}))=1 \text {, }
$$

as claimed.

The remaining part of the long exact sequence (12) now becomes the central column of the commutative diagram

$$
\begin{aligned}
& \begin{array}{c}
0 \\
H^{1}(\widehat{Z}, \mathscr{O}(T \widehat{Z}))
\end{array} \\
& \downarrow \\
& H^{0}(\mathscr{Q}, T \mathscr{B}) \quad \rightarrow \quad H^{1}(\mathscr{B}, \mathscr{K}(T \mathscr{B})) \rightarrow H^{1}(\mathscr{B}, \mathscr{O}(T \mathscr{B})) \rightarrow 0 \\
& \downarrow d f \quad \downarrow d f \quad \downarrow d f \\
& H^{0}\left(\mathscr{Q}, J^{1} \mathscr{O}(n, n, 2)\right) \rightarrow H^{1}\left(\mathscr{B}, \mathscr{K}^{2}(n, n, 2)\right) \rightarrow H^{1}(\mathscr{B}, \mathscr{O}(n, n, 2)) \rightarrow 0 \\
& H^{2}(\widehat{Z}, \mathscr{O}(T \widehat{Z}))
\end{aligned}
$$


with exact rows and columns. Since the cohomology

$$
H^{1}(\mathscr{B}, \mathscr{O}(T \mathscr{B}))=H^{1}\left(\mathbb{C P}_{1} \times \mathbb{C P}_{1}, \mathscr{O}(2-n, n-2) \oplus \mathscr{O}(n-2,2-n)\right)
$$

is carried by the subsheaf of vector fields of the form

$$
\mathscr{O}_{\mathbb{C P}_{1} \times \mathbb{C P}_{1}}(2-n, n-2) x \partial / \partial y+\mathscr{O}_{\mathbb{C P}_{1} \times \mathbb{C P}_{1}}(n-2,2-n) y \partial / \partial x,
$$

and since these are sent isomorphically by $d_{\pi} f=x d y+y d x$ to the subsheaf of $\mathscr{O}(n, n, 2)$ consisting of sections of the form

$$
\mathscr{O}_{\mathbb{C P}_{1} \times \mathbb{C P}_{1}}(2-n, n-2) x^{2}+\mathscr{O}_{\mathbb{C P}_{1} \times \mathbb{C P}_{1}}(n-2,2-n) y^{2},
$$

which in turn carries the cohomology

$$
H^{1}(\mathscr{B}, \mathscr{O}(n, n, 2))=H^{1}\left(\mathbb{C P}_{1} \times \mathbb{C P}_{1}, \mathscr{O}(2-n, n-2) \oplus \mathscr{O}(n-2,2-n)\right),
$$

the right-most vertical map of the above diagram, therefore, is an isomorphism, and we therefore have an induced diagram

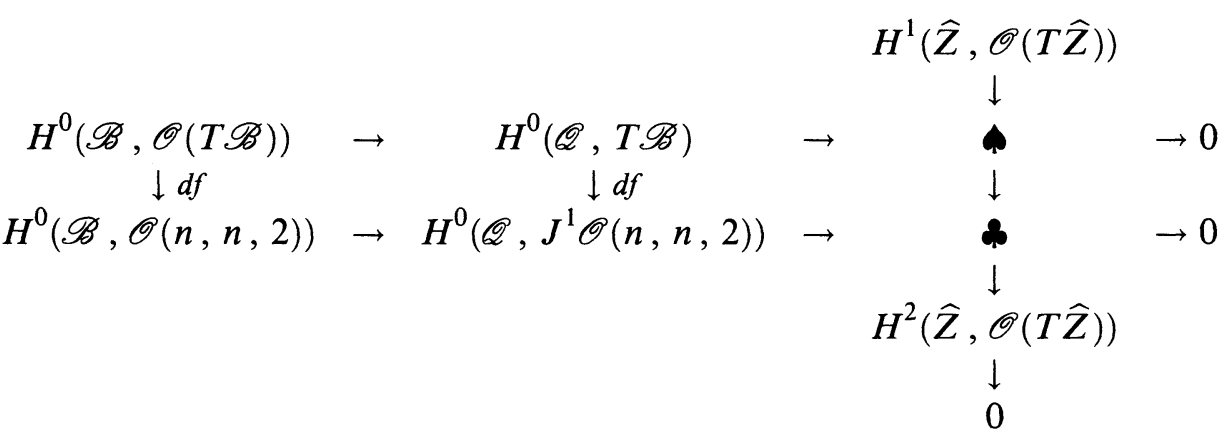

But at each point of $\mathscr{Q}, f$ has the form $x y+z w$ in appropriate local coordinates, so that $d f$ is of the form $x d y+y d x+z d w+w d z$; hence $d f$ maps $H^{0}(\mathscr{Q}, T \mathscr{B})$ isomorphically onto the subspace $H^{0}\left(\mathscr{Q}, \Omega_{\mathscr{B}}^{1} \mathscr{O}(n, n, 2)\right) \subset$ $H^{0}\left(\mathscr{Q}, J^{1} \mathscr{O}(n, n, 2)\right)$. On the other hand, the map

$$
\begin{aligned}
H^{0}(\mathscr{B}, \mathscr{O}(n, n, 2)) & \rightarrow H^{0}(\mathscr{Q}, \mathscr{O}(n, n, 2)) \\
& =H^{0}\left(\mathscr{Q}, J^{1} \mathscr{O}(n, n, 2)\right) / H^{0}\left(\mathscr{Q}, \Omega_{\mathscr{B}}^{1} \otimes \mathscr{O}(n, n, 2)\right)
\end{aligned}
$$

is surjective, since we can find elements $\alpha$ of $\Gamma\left(\mathbb{C P}_{1} \times \mathbb{C P}_{1}, \mathscr{O}(n, n)\right) \stackrel{t^{2}}{\hookrightarrow}$ $\Gamma(\mathscr{B}, \mathscr{O}(n, n, 2))$ that vanish at all points of $\mathscr{Q}$ except, say, $q_{k \ell}$, for instance by setting

$$
\alpha:=R_{\ell k}^{2} S_{\ell k}^{2} \prod_{j \neq k, \ell} P_{j},
$$

where $R_{\ell k}$ and $S_{\ell k}$ are sections of $\mathscr{O}(1,0)$ and $\mathscr{O}(0,1)$ that vanish at the conjugate point $q_{\ell k}=\rho\left(q_{k \ell}\right)$. It follows that the above map $\wedge \rightarrow \&$ is surjective, and $H^{2}(\widehat{Z}, \mathscr{O}(T \widehat{Z}))=0$, as claimed. Moreover, the kernels of 
$H^{0}(\mathscr{B}, \mathscr{O}(T \mathscr{B})) \rightarrow H^{0}(\mathscr{Q}, T \mathscr{B})$ and

$$
H^{0}(\mathscr{B}, \mathscr{O}(n, n, 2)) \rightarrow H^{0}\left(\mathscr{Q}, J^{1} \mathscr{O}(n, n, 2)\right)
$$

are $H^{0}(\mathscr{B}, \mathscr{K}(T \mathscr{B}))$ and $H^{0}\left(\mathscr{B}, \mathscr{K}^{2}(n, n, 2)\right)$, respectively, which, as we saw in (13) and (14) both turned out to be two-dimensional. Using our computations $(11)$ and $(9)$ of $h^{0}(\mathscr{B}, \mathscr{O}(T \mathscr{B}))$ and $h^{0}(\mathscr{B}, \mathscr{O}(n, n, 2))$, we therefore have

$$
\begin{aligned}
h^{1}(\widehat{Z}, \mathscr{O}(T \widehat{Z}))= & \operatorname{dim} \boldsymbol{\operatorname { d i m }} \mathbf{d} \\
= & {\left[h^{0}(\mathscr{Q}, T \mathscr{B})-h^{0}(\mathscr{B}, \mathscr{O}(T \mathscr{B}))+h^{0}(\mathscr{B}, \mathscr{K}(T \mathscr{B}))\right] } \\
& \quad-\left[h^{0}\left(\mathscr{Q}, J^{1} \mathscr{O}(n, n, 2)\right)-h^{0}(\mathscr{B}, \mathscr{O}(n, n, 2))\right. \\
& \left.+h^{0}\left(\mathscr{B}, \mathscr{K}^{2}(n, n, 2)\right)\right] \\
= & {[4 n(n-1)-(4 n+8)+2]-\left[5 n(n-1)-\left(n^{2}+6 n+2\right)+2\right] } \\
= & 3 n-6 .
\end{aligned}
$$

Remark. Notice that our construction of $Z$ depended on $3 n-6$ real parameters, this being the dimension of the space of generic configurations of $n$ points in hyperbolic 3 -space $\mathscr{H}^{3}$ modulo the action of the group $O_{+}^{\uparrow}(1,3)$ of hyperbolic isometries. We can generalize our construction of $\widehat{Z}$ slightly by dropping the reality condition on the polynomials $P_{1}, \ldots, P_{n}$, since this is only needed to specify the manner in which $\widehat{Z}$ is to be blown down to produce $Z$; in this form, our construction then essentially depends on $3 n-6$ complex parameters. It is not accidental that this is exactly the dimension of $H^{1}(\widehat{Z}, T \widehat{Z})$. Indeed, careful inspection of the surjection $H^{0}(\mathscr{Q}, T \mathscr{B}) \rightarrow H^{1}(\widehat{Z}, T \widehat{Z})$ and the corresponding diagram reveals that varying the polynomials $P_{1}, \ldots, P_{n}$ precisely generates $H^{1}(\widehat{Z}, T \widehat{Z}) \cong \mathbb{C}^{3 n-6}$, and this relation between the given parameter space and cohomology is essentially the Kodaira-Spencer map (cf. $\S 4$ ) of the corresponding deformation of $Z$.

The other dimensions of cohomology that we calculated also have direct interpretations. In particular, the fact that $h^{0}(\widehat{Z}, T \widehat{Z})=1$ amounts to saying that the corresponding self-dual manifolds $M \approx n \mathbb{C P}_{2}$ have one-dimensional isometry groups. On the other hand, the vanishing of $H^{2}(\widehat{Z}, T \widehat{Z})$ is related (cf. $\S 4)$ to the fact that every element of $H^{1}(\widehat{Z}, T \widehat{Z})$ is generated by a deformation of $\widehat{Z}$.

We now let $\mathscr{C}$ and $\overline{\mathscr{C}}$ denote the two curves in $Z$ obtained by blowing down $x=t=0$ and $y=t=0$ via $a$, and let $\Theta_{\mathscr{C} \overline{\mathscr{C}}} \subset \mathscr{O}(T Z)$ denote the sheaf of vector fields on $Z$ that are tangent to these two rational curves, that is, that have vanishing normal component along $\mathscr{C}$ and $\overline{\mathscr{C}}$. 
Proposition 2. Let $n \geq 3$. Then

$$
h^{j}\left(Z, \Theta_{\mathscr{C} \mathscr{C}}\right)=\left\{\begin{array}{cl}
1 & j=0, \\
3 n-6 & j=1, \\
0 & j \geq 2 .
\end{array}\right.
$$

Proof. For $j, k=1, \ldots, n, j \neq k$, let $\mathscr{C}_{j k} \subset Z$ denote the curves $\mathscr{C}_{j k}=$ $a\left[b^{-1}\left\{q_{j k}\right\}\right]$ and let $\mathscr{S}$ denote their union $\bigcup \mathscr{C}_{j k}$. Let $\check{\Theta} \subset \Theta_{\mathscr{C} \overline{\mathscr{C}}}$ denote the sheaf of vector fields that are tangent to the curves $\mathscr{C}_{j k}$, as well as to $\mathscr{C}$ and $\overline{\mathscr{C}}$. Then we have

$$
a_{*}^{j} \mathscr{O}(T \widehat{Z})= \begin{cases}\check{\Theta} & j=0, \\ 0 & j \neq 0,\end{cases}
$$

so that $H^{j}(Z, \check{\Theta})=H^{j}(\widehat{Z}, \mathscr{O}(T \widehat{Z})) \forall j$. On the other hand, there is an exact sequence

$$
0 \rightarrow \check{\boldsymbol{\Theta}} \rightarrow \boldsymbol{\Theta}_{\mathscr{C} \overline{\mathscr{C}}} \rightarrow \mathscr{O}_{\mathscr{S}}\left(\nu_{\mathscr{S}}\right) \rightarrow 0
$$

where $\nu_{\mathscr{S}} \rightarrow \mathscr{S}$ denotes the normal bundle of $\mathscr{S} \subset Z$, and so is isomorphic to $\mathscr{O}(-1) \oplus \mathscr{O}(-1)$ when restricted to $\mathscr{C}_{j k} \cong \mathbb{C P}_{1}$. Since all the cohomology of $\mathscr{O}_{\mathscr{S}}\left(\nu_{\mathscr{S}}\right)$ therefore vanish, the result follows.

Corollary 1. Let $n \geq 3$. Then

$$
h^{j}(Z, \mathscr{O}(T Z))=\left\{\begin{array}{cl}
1 & j=0 \\
7 n-14 & j=1 \\
0 & j \geq 2
\end{array}\right.
$$

Proof. There is an exact sequence

$$
0 \rightarrow \Theta_{\mathscr{C} \overline{\mathscr{C}}} \rightarrow \mathscr{O}(T Z) \rightarrow \mathscr{O}_{\mathscr{C} \overline{\mathscr{C}}}\left(\nu_{\mathscr{C} \overline{\mathscr{C}}}\right) \rightarrow 0
$$

where $\nu_{\mathscr{C} \overline{\mathscr{C}}} \rightarrow \mathscr{C} \cup \overline{\mathscr{C}}$ denotes the normal bundle of $\mathscr{C} \cup \overline{\mathscr{C}} \subset Z$. Since the normal bundles of $\mathscr{C} \cong \overline{\mathscr{C}} \cong \mathbb{C P}_{1}$ are isomorphic to $\mathscr{O}(1-n) \oplus \mathscr{O}(1-n)$, it follows that

$$
h^{j}\left(\mathscr{O}_{\mathscr{C} \overline{\mathscr{C}}}\left(\nu_{\mathscr{C} \overline{\mathscr{C}}}\right)\right)=\left\{\begin{array}{cc}
4 n-8 & j=1, \\
0 & j \neq 1 .
\end{array}\right.
$$

Using the above exact sequence, the result then follows from Proposition 2.

Remark. Let us observe that the above calculation gives

$$
\chi(Z, \mathscr{O}(T Z))=15-7 n
$$

for the index of the tangent bundle of $Z$. We can double-check this by use of the Hirzebruch-Riemann-Roch formula and Hitchin's formulas [13] for the Chern numbers of a twistor space. Indeed, for any compact complex 3-fold $Z$, 
one has

$$
\begin{aligned}
\chi(Z, & \mathscr{O}(T Z)) \\
& =\int_{Z} T d(Z) \operatorname{ch}(T Z) \\
& =\int_{Z}\left[1+\frac{c_{1}}{2}+\frac{c_{1}^{2}+c_{2}}{13}+\frac{c_{1} c_{2}}{24}\right]\left[3+c_{1}+\frac{c_{1}^{2}-2 c_{2}}{2}+\frac{c_{1}^{3}-3 c_{1} c_{2}+3 c_{3}}{6}\right] \\
& =\frac{c_{1}^{3}-3 c_{1} c_{2}+3 c_{3}}{6}+\frac{c_{1}^{3}-2 c_{1} c_{2}}{4}+\frac{c_{1}^{3}+c_{1} c_{2}}{13}+\frac{c_{1} c_{2}}{8} \\
& =\frac{c_{1}^{3}}{2}-\frac{19 c_{1} c_{2}}{24}+\frac{c_{3}}{2} .
\end{aligned}
$$

On the other hand, if $Z$ is the twistor space of a compact self-dual 4-manifold $M$, it follows that

$$
c_{1}^{3}=16(2 \chi-3 \tau), \quad c_{1} c_{2}=12(\chi-\tau), \quad c_{3}=2 \chi
$$

where $\chi$ and $\tau$ are the Euler characteristic and signature of $M$, respectively. For $M \approx n \mathbb{C P}_{2}$, we must therefore have

$$
\begin{aligned}
\chi(Z, \mathscr{O}(T Z)) & =8(2 \chi-3 \tau)-\frac{19}{2}(\chi-\tau)+\chi \\
& =\frac{1}{2}(15 \chi-29 \tau) \\
& =\frac{1}{2}(15(2+n)-29 n) \\
& =15-7 n,
\end{aligned}
$$

precisely in accordance with our calculations. Indeed, we could have used just such an index calculation to avoid calculating, say, $h^{1}(\widehat{Z}, \mathscr{O}(T \widehat{Z}))$. We opted not to do so, however, both in order to maintain the concrete spirit of the proof and to provide an added check on the consistency of our constructions and calculations.

\section{DEFORMATION THEORY}

Let $\mathscr{U}$ be a complex k-manifold, and let $o \in \mathscr{U}$ be a chosen base point, which we will refer to as the origin of $\mathscr{U}$. Recall that a family of compact complex $n$-manifolds over $\mathscr{U}$ then means a complex $(n+k)$-manifold $\mathscr{Z}$, together with a proper holomorphic submersion $\varpi: \mathscr{Z} \rightarrow \mathscr{U}$ onto $\mathscr{U}$. For each $u \in \mathscr{U}$, we set $Z_{u}:=\varpi^{-1}(t)$, and call $Z:=Z_{o}$ the central fiber. Such a family $\mathscr{Z}$, or, more loosely, such a collection $\left\{Z_{u}\right\}$ of compact complex manifolds, will be called a deformation of the central fiber $Z$. $\mathscr{U}$ is called the parameter space of the deformation. We are generally only interested in the germ of a deformation at the central fiber; for practical purposes one may therefore, of course, take $\mathscr{U}$ to simply be a ball in $\mathbb{C}^{k}$ centered at $o=\overrightarrow{0}$. 
If we restrict the tangent bundle $T \mathscr{Z}$ of the total space of such a family to the central fiber $Z$, we get a short exact sequence

$$
\left.0 \rightarrow T Z \rightarrow T \mathscr{Z}\right|_{Z} \rightarrow \varpi^{*} T_{o} \mathscr{U} \rightarrow 0
$$

involving the tangent bundle of $Z$ and the (trivial) normal bundle of $Z \subset$ $\mathscr{U}$. From the associated long exact sequence we therefore have a connecting homomorphism

$$
\delta: H^{0}\left(Z, \mathscr{O}\left(\varpi^{*} T_{o} \mathscr{U}\right)\right) \rightarrow H^{1}(Z, \mathscr{O}(T Z)) .
$$

Since $H^{0}\left(Z, \mathscr{O}\left(\varpi^{*} T_{o} \mathscr{U}\right)\right)=T_{o} \mathscr{U}$, this may be reinterpreted as a linear map

$$
k s: T_{o} \mathscr{U} \rightarrow H^{1}(Z, \mathscr{O}(T Z)),
$$

known as the Kodaira-Spencer map of the family $\mathscr{Z}$ at $o$, and is precisely a measure of the degree to which $\varpi: \mathscr{Z} \rightarrow \mathscr{U}$ fails to be trivial to first order at $t=o$. If the ks happens to be an isomorphism, we say that the family $\mathscr{Z}$ is versal at $t=o$. In this case, of course, the dimension of $\mathscr{U}$ is necessarily $h^{1}(Z, \mathscr{O}(T Z))$.

Now if $\mathrm{Z}$ is any given compact complex manifold such that $H^{2}(Z, \mathscr{O}(T Z))=$ 0 , the main theorem of Kodaira-Spencer theory $[17,18]$ tells us that there exists a versal deformation of $Z$. Moreover, this versal deformation is germunique at the central fiber, in the sense that any two such versal families are isomorphic on the level of germs at $Z$, and the derivative at the base point $o$ of the associated map between their parameter spaces is uniquely determined. However, there is in general no preferred isomorphism between the germs of two versal deformations.

Relative versions of the theory are also available. For instance, if $Z$ is a compact complex manifold and $D \subset Z$ is a smooth hypersurface, let $\Theta_{D} \subset$ $\mathscr{O}(T Z)$ denote the subsheaf of vector fields that are tangent to $D$, i.e., that have vanishing normal component along $D$. If $H^{2}\left(Z, \Theta_{D}\right)=0$, then there exists a versal deformation of the pair $(Z, D)$, meaning a family $\mathscr{Z} \rightarrow \mathscr{U}$ equipped with a subfamily $\mathscr{D} \subset \mathscr{Z}$ such that the relative Kodaira-Spencer map $T_{o} \mathscr{U} \rightarrow H^{1}\left(Z, \Theta_{D}\right)$ is an isomorphism; here the relative Kodaira-Spencer map is by definition the connecting homomorphism induced by the exact sequence

$$
0 \rightarrow \Theta_{D} \rightarrow \widehat{\Theta}_{\mathscr{D}} \rightarrow \varpi^{*} T_{o} \mathscr{U} \rightarrow 0
$$

where $\widehat{\Theta}_{\mathscr{D}}$ is the sheaf of holomorphic sections of $\left.T \mathscr{Z}\right|_{Z}$ that are tangent to $\mathscr{D}$ along $D$. In the same spirit, if $D$ and $\bar{D}$ are two hypersurfaces of $Z$ that meet transversely, there is a versal family for the triple $(Z, D, \bar{D})$ provided that $H^{2}\left(Z, \Theta_{D \bar{D}}\right)=0$, where $\Theta_{D \bar{D}}=\Theta_{D} \cap \Theta_{\bar{D}}$.

If $Z$ is any compact twistor space, and if $D \subset Z$ is any reduced compact complex surface, we define the degree of $D$ to be the number of points in which $D$ intersects a generic twistor line; equivalently, the degree is given by $\int_{L} c_{1}[D]$, where $[D]$ denotes the divisor line bundle of $D$ and $L$ is any twistor 
line. If $D \subset Z$ is a surface of degree 1 , and if $L$ is any twistor line, then $D$ either contains $L$ or meets it in precisely one point. It then follows that $D \cap \bar{D}$ is a union of twistor lines, where $\bar{D}$ is the image of $D$ under the real structure $\sigma: Z \rightarrow Z$. In this section we will consider deformations of the triple $(Z, D, \bar{D})$, where $Z$ is one of the twistor spaces constructed on page 293, and where $D$ is a degree 1 surface.

The surfaces we will consider are constructed as follows: let $\pi_{1}: \widetilde{Z} \rightarrow \mathbb{C P}_{1}$ denote the first factor of the projection $\pi: \mathscr{B} \rightarrow \mathbb{C P}_{1} \times \mathbb{C P}_{1}$ restricted to $\widetilde{Z} \subset \mathscr{B}$, and let $\left[z_{0}, z_{1}\right] \in \mathbb{C P}_{1}$ denote a point not in the image of $\mathscr{Q}$ under $\pi_{1}$. Then $\widetilde{D}:=\pi_{1}^{-1}\left\{\left[z_{0}, z_{1}\right]\right\}$ is a smooth hypersurface, and $D:=a\left(b^{-1}(\widetilde{D})\right)$ is then the desired surface of degree 1 . We analogously construct a surface $\bar{D}:=a\left(b^{-1}(\widetilde{\bar{D}})\right)$ from $\widetilde{\bar{D}}:=\pi_{2}^{-1}\left\{\left[\overline{z_{0}, z_{1}}\right]\right\}$, where $\pi_{2}$ denotes the second factor of $\left.\pi\right|_{\tilde{Z}}: \widetilde{Z} \rightarrow \mathbb{C P}_{1} \times \mathbb{C P}_{1}$.

As a complex surface, $D$ is the blow-up of $\mathbb{C P}_{2}$ at $n$ distinct points arranged along a projective line whose proper transform is $\mathscr{C}$. Indeed, by virtue of the projection $\pi_{2}$ to $\mathbb{C P}_{1}$ with which it is equipped by construction, $\widetilde{D}$ is a rational ruled surface. It contains two rational curves $x=t=0$ and $y=t=0$, respectively with self-intersection numbers $1-n$ and -1 , that are sections of $\pi_{2}: \widetilde{D} \rightarrow \mathbb{C P}_{1}$; and there are exactly $n$ singular fibers of this projection, each containing a single, isolated normal crossing corresponding to a root of $\prod P_{j}$. On the other hand, $a$ blows down the $(-1)$-curve $y=t=0$, but is otherwise trivial on $b^{-1}(\widetilde{D})$. This shows that the only exceptional curves in $D$ are $\mathscr{C}$ and $n$ exceptional curves of the first kind, each of which meets $\mathscr{C}$. Blowing down $D$ along the latter $n$ curves then produces a minimal surface containing a curve of self-intersection 1 , so that the image of $\mathscr{C}$ is a line in $\mathbb{C P}_{2}$, as claimed. Applying $\sigma$, we similarly conclude that $\bar{D}$ is isomorphic to $\mathbb{C P}_{2}$ blown up at $n$ points arranged along a line whose proper transform is $\overline{\mathscr{C}}$.

We now let $\Theta_{D \bar{D}} \subset \mathscr{O}(T Z)$ denote the sheaf of vector fields on $Z$ that are tangent to both $D$ and $\bar{D}$.

Lemma 1. The natural restriction map

$$
H^{1}\left(D, \mathscr{O}\left(\nu_{D}\right)\right) \rightarrow H^{1}\left(\mathscr{C}, \mathscr{O}\left(\left.\nu_{D}\right|_{\mathscr{C}}\right)\right)
$$

is an isomorphism.

Proof. The normal bundle of $b^{-1}[\widetilde{D}] \subset \widehat{Z}$, like that of $\widetilde{D} \subset \widetilde{Z}$, is trivial. Now $a$ blows down the surfaces $x=t=0$ to $\mathscr{C}$ and $y=t=0$ to $\overline{\mathscr{C}}$, in the process sending a nonvanishing section of the normal bundle of $b^{-1}[\widetilde{D}]$ to one that vanishes precisely at $\mathscr{C}$. It follows that the normal bundle $\nu_{D}$ of $D \subset Z$ is exactly the divisor line bundle of $\mathscr{C} \subset D$. We therefore have a short exact sequence

$$
0 \rightarrow \mathscr{O} \rightarrow \mathscr{O}\left(\nu_{D}\right) \rightarrow \mathscr{O}_{\mathscr{C}}\left(\left.\nu_{D}\right|_{\mathscr{C}}\right) \rightarrow 0
$$

of sheaves on $D$. Since $D$ is a rational surface, $H^{1}(D, \mathscr{O})=H^{2}(D, \mathscr{O})=0$, and the result follows from the long exact sequence induced by (16). 
With this observation in hand, we are now in a position to prove the following vanishing theorem:

Proposition 3. $H^{2}\left(Z, \Theta_{D \bar{D}}\right)=0$.

Proof. The quotient map $\left.\nu_{C} \rightarrow \nu_{D}\right|_{\mathscr{C}}$ induces a surjection

$$
H^{1}\left(\mathscr{C}, \mathscr{O}\left(\nu_{\mathscr{C}}\right)\right) \rightarrow H^{1}\left(\mathscr{C}, \mathscr{O}\left(\left.\nu_{D}\right|_{\mathscr{C}}\right)\right)
$$

because $H^{2}$ of $\mathscr{C}$ with coefficients in the normal bundle of $\mathscr{C} \subset D$ vanishes for dimensional reasons. Similar reasoning applies to $\overline{\mathscr{C}}$ and $\bar{D}$. Since Proposition 2 asserts that $H^{2}\left(Z, \Theta_{\mathscr{C} \overline{\mathscr{C}}}\right)=0$, the restriction map

$$
H^{1}(Z, \mathscr{O}(T Z)) \rightarrow H^{1}\left(\mathscr{C}, \mathscr{O}\left(\nu_{\mathscr{C}}\right)\right) \oplus H^{1}\left(\overline{\mathscr{C}}, \mathscr{O}\left(\nu_{\overline{\mathscr{C}}}\right)\right)
$$

is surjective, and we therefore conclude that the natural map

$$
H^{1}(Z, \mathscr{O}(T Z)) \rightarrow H^{1}\left(\mathscr{C}, \mathscr{O}\left(\left.\nu_{D}\right|_{\mathscr{C}}\right)\right) \oplus H^{1}\left(\overline{\mathscr{C}}, \mathscr{O}\left(\left.\nu_{\bar{D}}\right|_{\overline{\mathscr{C}}}\right)\right)
$$

is onto. But this map factors as the composition of the restriction map

$$
r: H^{1}(Z, \mathscr{O}(T Z)) \rightarrow H^{1}\left(D, \mathscr{O}\left(\nu_{D}\right)\right) \oplus H^{1}\left(\bar{D}, \mathscr{O}\left(\nu_{\bar{D}}\right)\right)
$$

and the restriction map

$$
H^{1}\left(D, \mathscr{O}\left(\nu_{D}\right)\right) \oplus H^{1}\left(\bar{D}, \mathscr{O}\left(\nu_{\bar{D}}\right)\right) \rightarrow H^{1}\left(\mathscr{C}, \mathscr{O}\left(\left.\nu_{D}\right|_{\mathscr{C}}\right)\right) \oplus H^{1}\left(\overline{\mathscr{C}}, \mathscr{O}\left(\left.\nu_{\bar{D}}\right|_{\overline{\mathscr{C}}}\right)\right) .
$$

By Lemma 1 and its barred analogue, the latter is an isomorphism, and we therefore conclude $r$ is an epimorphism. The claim, therefore, follows from the long exact sequence

$$
\begin{aligned}
\cdots & \rightarrow H^{1}(Z, \mathscr{O}(T Z)) \stackrel{r}{\rightarrow} H^{1}\left(D, \mathscr{O}\left(\nu_{D}\right)\right) \oplus H^{1}\left(\bar{D}, \mathscr{O}\left(\nu_{\bar{D}}\right)\right) \\
& \rightarrow H^{2}\left(Z, \Theta_{D \bar{D}}\right) \rightarrow H^{2}(Z, \mathscr{O}(T Z)) \rightarrow \cdots,
\end{aligned}
$$

and the vanishing of $H^{2}(Z, \mathscr{O}(T Z))$ proved in Corollary 1 .

This immediately implies:

Theorem 1. There exists a smooth versal deformation of $(Z, D, \bar{D})$, where $Z$ is any of the twistor spaces constructed in $\S 2$ and where $D$ and $\bar{D}$ are a conjugate pair of degree 1 divisors as described above. Moreover, the complex dimension of the parameter space of this family is $5 n-8$.

Proof. It remains only to compute $h^{1}\left(Z, \Theta_{D \bar{D}}\right)$. For this we use the exact sequence

$$
\begin{aligned}
\cdots & \rightarrow H^{0}(Z, \mathscr{O}(T Z)) \rightarrow H^{0}\left(D, \mathscr{O}\left(\nu_{D}\right)\right) \oplus H^{0}\left(\bar{D}, \mathscr{O}\left(\nu_{\bar{D}}\right)\right) \\
& \rightarrow H^{1}\left(Z, \Theta_{D \bar{D}}\right) \rightarrow H^{1}(Z, \mathscr{O}(T Z)) \stackrel{r}{\rightarrow} H^{1}\left(D, \mathscr{O}\left(\nu_{D}\right)\right) \oplus H^{1}\left(\bar{D}, \mathscr{O}\left(\nu_{\bar{D}}\right)\right) .
\end{aligned}
$$

From the exact sequence (16), together with the fact that $H^{0}\left(\mathscr{C},\left.\nu_{D}\right|_{\mathscr{C}}\right)=$ $H^{0}\left(\mathbb{C P}_{1}, \mathscr{O}(2-n)\right)=0$, we conclude that $h^{0}\left(D, \mathscr{O}\left(\nu_{D}\right)\right)=1 ;$ similarly, 
$h^{0}\left(\bar{D}, \mathscr{O}\left(\nu_{\bar{D}}\right)\right)=1$. On the other hand, the natural $\mathbb{C}_{*}$-action

$$
\begin{aligned}
\widetilde{\Phi}: \mathbb{C}_{*} \times \widetilde{Z} & \rightarrow \widetilde{Z} \\
(\lambda,[x, y, t]) & \mapsto\left[\lambda x, \lambda^{-1} y, t\right]
\end{aligned}
$$

on $\tilde{Z}$ gives rise to an action $\Phi: \mathbb{C}_{*} \times Z \rightarrow Z$ that generates $H^{0}(Z, \mathscr{O}(T Z)) \cong \mathbb{C}$; since and $\Phi$ preserves $\widetilde{D}$, we may therefore observe that the map $H^{0}(Z, \mathscr{O}(T Z)) \rightarrow H^{0}\left(D, \mathscr{O}\left(\nu_{D}\right)\right) \oplus H^{0}\left(\bar{D}, \mathscr{O}\left(\nu_{\bar{D}}\right)\right)$ vanishes. From the surjectivity of $r$ we thus conclude that

$$
\begin{aligned}
h^{1}\left(Z, \Theta_{D \bar{D}}\right)= & h^{1}(Z, \mathscr{O}(T Z))-\left[h^{1}\left(D, \mathscr{O}\left(\nu_{D}\right)\right)+h^{1}\left(\bar{D}, \mathscr{O}\left(\nu_{\bar{D}}\right)\right)\right] \\
& +\left[h^{0}\left(D, \mathscr{O}\left(\nu_{D}\right)\right)+h^{0}\left(\bar{D}, \mathscr{O}\left(\nu_{\bar{D}}\right)\right)\right] \\
= & h^{1}(Z, \mathscr{O}(T Z))-2 h^{1}\left(\mathscr{C},\left.\nu_{D}\right|_{\mathscr{C}}\right)+2 \\
= & (7 n-14)-2(n-2)+2=5 n-8 .
\end{aligned}
$$

Of course, we could have chosen just to deform $Z$ alone, rather than deforming the triple $(Z, D, \bar{D})$. In this case, the parameter space of the versal deformation would have instead had dimension $7 n-14$. Or, had we chosen to deform the triple $(Z, \mathscr{C}, \overline{\mathscr{C}})$, the family would have had dimension $3 n-6$, and actually would have simply corresponded to varying the sections $P_{1}, \ldots, P_{n} \in \Gamma\left(\mathbb{C P}_{1} \times \mathbb{C P}_{1}, \mathcal{O}(1,1)\right)$ in equation (2).

We now turn to the study of the effect of these deformations on the intrinsic structure of the complex surface $D$. Let $\mathscr{T}_{D} \subset \mathscr{O}(T D)$ denote the sheaf of tangent fields on $D$ that are tangent to the twistor line $L$. Let $\nu_{\mathscr{C} D}$ denote the normal bundle of $\mathscr{C} \subset D$. We then have a natural map $\Theta_{D \bar{D}} \rightarrow \mathscr{O}_{\mathscr{E}}\left(\nu_{\mathscr{E} D}\right)$ that factors through the natural map $\Theta_{D \bar{D}} \rightarrow \mathscr{T}_{D}$, similarly, for $\bar{D}$ and $\overline{\mathscr{C}}$. Using the fact that $D$ is isomorphic to $\mathbb{C P}_{2}$ blown up at $n$ collinear points, one may check that $h^{1}(D, \mathscr{O}(T D))=2 n-5$, so that $h^{1}\left(D, \mathscr{T}_{D}\right)=2 n-4$. But $h^{1}\left(\mathscr{C}, \mathscr{O}_{\mathscr{C}}\left(\nu_{\mathscr{C} D}\right)\right)=h^{1}\left(\mathbb{C P}_{1}, \mathscr{O}(1-n)\right)=h^{0}\left(\mathbb{C P}_{1}, \mathscr{O}(n-3)\right)=n-2$, and the map $H^{1}\left(D, \mathscr{T}_{D}\right) \rightarrow H^{1}\left(\mathscr{C}, \mathscr{O}\left(\nu_{\mathscr{C} D}\right)\right)$ is surjective, with $(n-2)$-dimensional kernel corresponding to choices of $n$ preferred points on $\mathscr{C}-L$. From the surjectivity of $H^{1}(Z, \mathscr{O}(T Z)) \rightarrow H^{1}\left(\mathscr{C}, \nu_{\mathscr{C}}\right) \oplus H^{1}\left(\overline{\mathscr{C}}, \nu_{\mathscr{\mathscr { C }}}\right)$, one now deduces that $H^{1}\left(Z, \Theta_{D \bar{D}}\right) \rightarrow H^{1}\left(\mathscr{C}, \mathscr{O}\left(\nu_{\mathscr{C} D}\right)\right) \oplus H^{1}\left(\frac{\mathscr{C}}{\mathscr{C}}, \mathscr{O}\left(\nu_{\overline{\mathscr{C}} D}\right)\right)$ is also surjective. From our freedom to move the $2 n$ given points on $\mathscr{C}$ and $\overline{\mathscr{C}}$ independently by varying the polynomials $P_{1}, \ldots, P_{n}$, we thus conclude that

$$
H^{1}\left(Z, \Theta_{D \bar{D}}\right) \rightarrow H^{1}\left(D, \mathscr{T}_{D}\right) \oplus H^{1}\left(\bar{D}, \mathscr{T}_{\bar{D}}\right)
$$

is surjective. In other words,

Proposition 4. All small deformations of $(D, L) \amalg(\bar{D}, L)$ are induced by deformations of $(Z, D, \bar{D})$. 
Now all the families we have considered so far are merely versal, not univer$\mathrm{sal}$; as we will see more clearly in a moment, there are many distinct values of $u \in \mathscr{U}$ such that the corresponding fibers $Z_{u}$ are isomorphic. This is related to the fact that the central fiber $Z$ carries a nontrivial vector field, whereas, as we will see in a moment, the typical fiber $Z_{u}$ does not. We can get around this problem by the following trick: let $L \cong \mathbb{C P}_{1}$ denote the line $D \cap \bar{D} \subset Z$, and let $p \in L$ be any point at which the generator of $h^{0}\left(Z, \Theta_{D \bar{D}}\right)=h^{0}(Z, \mathscr{O}(T Z)) \cong \mathbb{C}$ is nonzero; this is possible because this generator vanishes on $L$ precisely at the two points $L \cap \mathscr{C}$ and $L \cap \overline{\mathscr{C}}$. We may then consider the deformation theory of the quadruple $(Z, D, \bar{D}, p)$, meaning that for each point $u \in \mathscr{U}$ of the parameter space we require that the corresponding fiber $Z_{u}$ be equipped with not only a pair of transverse hypersurfaces $D_{u}$ and $\bar{D}_{u}$, but also with a "base point" $p_{u} \in L_{u}:=\left(D_{u} \cap \bar{D}_{u}\right)$ as well. The relevant deformation theory is then controlled by the cohomology of the sheaf $\Theta_{D \bar{D} p}$ consisting of elements of $\Theta_{D \bar{D}}$ that vanish at $p$. From the exact sequence

$$
0 \rightarrow \Theta_{D \bar{D} p} \rightarrow \Theta_{D \bar{D}} \rightarrow T_{p} L \rightarrow 0,
$$

we conclude that the dimensions of the relevant cohomology groups are then given by

$$
h^{j}\left(Z, \Theta_{D \bar{D} p}\right)=\left\{\begin{array}{cc}
5 n-8 & j=1, \\
0 & j \neq 1 .
\end{array}\right.
$$

There is therefore a versal deformation of the quadruple $(Z, D, \bar{D}, p)$. Moreover, by semicontinuity and the fact that the index must be constant, we have

$$
h^{1}\left(Z_{u}, \Theta_{D \bar{D} p}\right)=5 n-8
$$

for $u$ in a neighborhood of the base point $o$, and we might as well take this neighborhood to be all of the $(5 n-8)$-dimensional parameter space $\mathscr{U}$. Thus $\varpi_{*}^{1} \Theta_{D \bar{D}} p$ becomes a holomorphic vector bundle on $\mathscr{U}$, and the Kodaira-Spencer map becomes a holomorphic vector-bundle-valued 1 -form on $\mathscr{U}$ with maximal rank at the origin [10]. We conclude that our family is versal for all values of $u \in$ $\mathscr{U}$, and so is universal; i.e., any deformation of the quadruple $(Z, D, \bar{D}, p)$ is induced by a unique map from its parameter space into $\mathscr{U}$. Moreover, since the connected component of the automorphism group of $(Z, D, \bar{D}, p)$ is trivial, it follows that such a map of parameter spaces lifts uniquely to a map of the total spaces of the relevant families.

Recall that the divisor $D$ in the central fiber is isomorphic to $\mathbb{C P}_{2}$ blown up at $n$ distinct points arranged along a line whose proper transform is $\mathscr{C}$. Let us now choose a "marking" consisting of the homology classes on $D$ of two of the exceptional divisors associated with these blown-up points. We then have corresponding classes on $\bar{D}$. For each fiber $Z_{u}$ of our universal family we then have unique exceptional curves in the corresponding homology classes of $D_{u}$ and $\bar{D}_{u}$, and by blowing these down, joining the resulting points by projective lines, and then intersecting these lines with $L_{u}$, we produce pairs $\left(0_{u}, \infty_{u}\right)$ 
of distinct preferred points in $L_{u}$. The collection of fibers $L_{u}-\left\{0_{u}, \infty_{u}\right\}$ thus yields a principal holomorphic $\mathbb{C}_{*}$-bundle over $\mathscr{U}$ depending only on our choice of marking. Our base points may now be varied by applying the resulting $\mathbb{C}_{*}$-action to $p_{u}$, and this base point change then induces a $\mathbb{C}_{*}$-action

$$
\begin{array}{ccc}
\mathbb{C}_{*} \times \mathscr{Z} & \stackrel{\widehat{\varphi}}{\longrightarrow} & \mathscr{Z} \\
1 \times \varpi \downarrow & & \downarrow \varpi \\
\mathbb{C}_{*} \times \mathscr{U} & \stackrel{\varphi}{\longrightarrow} & \mathscr{U}
\end{array}
$$

by virtue of the universal property. The functor that "forgets" $p_{u}$ now identifies the universal family of $(Z, D, \bar{D}, p)$ with the versal family for $(Z, D, \bar{D})$, and so, having imposed a choice of marking, changing the base point $p$ of the central fiber induces a $\mathbb{C}_{*}$-action on the total space of the versal family. We will see in a moment that the action $\varphi$ on the parameter space $\mathscr{U}$ is actually a nontrivial action; indeed, its fixed point set will turn out to exactly be the $(3 n-6)$-dimensional family constructed in $\S 2$.

The fact that the versal family of $(Z, D, \bar{D})$ may be made universal by introducing a base point $p$ becomes particularly helpful when we wish to consider real structures. Let us observe that we may choose our base point $p$ to satisfy

$$
\varphi(-1, p)=\sigma(p)
$$

indeed, this just constrains $p$ to lie on an "equator" $\mathbb{R P}_{1} \subset \mathbb{C P}_{1}$ in $D \cap \bar{D}$. If we let $\bar{Z}$ and $\overline{\mathscr{U}}$ denote $\mathscr{Z}$ and $\mathscr{U}$ endowed with their conjugate complex structures and equip $\overline{\mathscr{Z}}$ with the family of base points $\varphi\left(-1, p_{u}\right)$, while simultaneously interchanging the order of the hypersurfaces $D_{u}$ and $\bar{D}_{u}, \varpi$ may be viewed as a deformation of $(\bar{Z}, \bar{D}, D, \sigma(p))$, so that $\sigma$ becomes a holomorphic identification of the central fibers of $\bar{Z} \rightarrow \overline{\mathscr{U}}$ and $\mathscr{Z} \rightarrow \mathscr{U}$, respecting the choices of hypersurfaces and base points. By the universal property, the antiholomorphic automorphism $\sigma: Z \rightarrow Z$ (i.e., the holomorphic map $\sigma: \bar{Z} \rightarrow Z$ ) automatically extends to an antiholomorphic automorphism

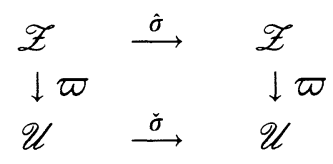

of the universal family $\varpi: \mathscr{Z} \rightarrow \mathscr{U}$ of $(Z, D, \bar{D}, p)$. By construction,

$$
\begin{aligned}
\hat{\sigma}\left(L_{u}\right) & =L_{\check{\sigma}(u)} \\
\hat{\sigma}\left(\varphi\left(-1, p_{u}\right)\right) & =p_{\check{\sigma}(u)}, \\
\hat{\sigma}\left(0_{u}\right) & =\infty_{\check{\sigma}(u)}, \\
\hat{\sigma}\left(\infty_{u}\right) & =0_{\check{\sigma}(u)} .
\end{aligned}
$$

Since $\hat{\sigma}$ is antiholomorphic, it follows that $\hat{\sigma}\left(p_{u}\right)=\varphi\left(-1, p_{\check{\sigma}(u)}\right)$; i.e., $\hat{\sigma}^{-1}\left(\varphi\left(-1, p_{u}\right)\right)=p_{\check{\sigma}^{-1}(u)}$. Also by construction, $\hat{\sigma}$ interchanges the divisor systems $\left\{D_{u}\right\}$ and $\left\{\bar{D}_{u}\right\}$. Since $\sigma=\sigma^{-1}$, and since $\hat{\sigma}$ and $\hat{\sigma}^{-1}$ have the same 
effect on base points and divisors, it now follows from the universal property that $\hat{\sigma}=\hat{\sigma}^{-1}$, and $\hat{\sigma}$ is thus an antiholomorphic involution. The fixed-point set $U \subset \mathscr{U}$ of $\check{\sigma}$, therefore, is a real-analytic manifold of dimension $5 n-8$. For each $u \in U$, the corresponding fiber $Z_{u}$ then has an involution $\sigma$ obtained by restricting $\hat{\sigma}$ to the fiber; this real structure is fixed-point-free and interchanges the divisors $D_{u}$ and $\bar{D}$. And although we shall not need this fact explicitly, one may also observe that the restriction $\mathbf{Z}$ of the family $\mathscr{Z}$ to $\mathbf{U}$ is now universal for $(Z, \sigma, D, p)$. To summarize:

Theorem 2. For each $n \geq 3$, there is $(5 n-8)$-parameter real versal family for the triple $(Z, \sigma, D)$. Moreover, this family is universal for the quadruple $(Z, \sigma$, $D, p)$, where the base point $p$ of any deformation is required to lie in the equator $S^{1} \subset D \cap \sigma(D)$ associated with a homology marking.

Remark. There is a rather more natural explanation for the existence of such real versal families. While the quotient $Z / \sigma$ is not a complex manifold it is a smooth manifold, and comes equipped with a pseudogroup structure in which the transition functions between coordinate charts are either holomorphic or antiholomorphic; thus $Z / \sigma$ has a structure analogous to that of a nonorientable surface with conformal structure. All of Kodaira-Spencer theory goes through for such objects, the relevant cohomology being that of the sheaf of real-holomorphic vector fields tangent to the appropriate submanifolds. But the cohomology of $Z / \sigma$ with coefficients in this sheaf is just a real form for the cohomology of $Z$ with coefficients in the sheaf of holomorphic vector fields tangent to the relevant submanifolds.

\section{SCALAR-Flat KäHLER SURFACES}

Having deformed our model twistor spaces $Z$ relative to a divisor in $\S 4$, let us now, in the spirit of [25], study the corresponding deformations of the associated half-conformally-flat 4-dimensional geometry. We thus consider the fibers $Z_{u}$ of the real versal deformation $Z \rightarrow U$ of $(Z, \sigma, D)$ constructed in Theorem 2 , remembering that each comes equipped with an involution $\sigma_{u}: Z_{u} \rightarrow Z_{u}$ without fixed points and a divisor $D_{u}$. The central fiber $Z$ is foliated by rational curves of normal bundle $\mathscr{O}(1) \oplus \mathscr{O}(1)$ that are $\sigma$-invariant. For each $u \in U$, let $\mathscr{M}_{u}$ denote the space of rational curves in $Z_{u}$ with normal bundle $\mathscr{O}(1) \oplus \mathscr{O}(1)$. By Kodaira's stability theorem [16], this is a complex 4-manifold for each $u$. The involution $\sigma_{u}$ sends each such curve to another one of the same kind, so that there is an induced involution $\sigma_{u \natural}: \mathscr{M}_{u} \rightarrow \mathscr{M}_{u}$. Let $\widehat{M}_{u}$ denote the fixed-point set of $\sigma_{u \natural}$; this is then a real-analytic 4-manifold for each $u$. The connected component $M=M_{o}$ of $\widehat{M}_{o}$ having the line $L:=D \cap \bar{D}$ as an element is a compact manifold, diffeomorphic to $n \mathbb{C P}_{2}$, and by shrinking $\mathbf{U}$ we may thus assume that the component $M_{u}$ of $\widehat{M}_{u}$ containing $L_{u}:=D_{u} \cap \mathscr{B} D_{u}$ is also compact-and thus of course also diffeomorphic to $n \mathbb{C P}_{2}$. By construction, 
$M_{u}$ comes equipped with a bundle $F_{u} \rightarrow M_{u}$ of $\mathbb{C P}_{1}$ 's, and a natural map $F_{u} \rightarrow Z_{u}$, so that the fiber of $F_{u}$ over $p \in M_{u}$ projects to the rational curve in $Z_{u}$ represented by $p$. This is automatically a local diffeomorphism because no $\sigma_{u}$-invariant section of the normal bundle of one of our rational curves can have a zero. Since $F_{u}$ and $Z_{u}$ are both compact, $F_{u} \rightarrow Z_{u}$ is a covering map, and hence a diffeomorphism because $Z_{u}$ is simply connected. This then induces a twistor fibration $\wp_{u}: Z_{u} \rightarrow M_{u}$ and induces a half-conformally-flat conformal metric on $M_{u}$.

The divisor $D_{u}$ meets every fiber of the twistor fibration $\wp_{u}$ transversely in one point, with the sole exception of $L_{u} \subset D_{u}$. The map $\wp_{u b}: D_{u} / L_{u} \rightarrow M_{u}$, therefore, is an orientation-reversing diffeomorphism $n \overline{\mathbb{C P}}_{2} \rightarrow n \mathbb{C P}_{2}$. If we let $\ell_{u} \in M_{u}$ denote the image of $L_{u}$ via $\wp_{u}$, we therefore conclude that $M_{u}-\left\{\ell_{u}\right\}$ is identified with an $\mathrm{n}$-fold blowup of $\mathbb{C}^{2}$ by $\wp_{u}$. Moreover, the conformal metric of $M_{u}$ becomes Hermitian with respect to this complex structure. Indeed, there is a choice of conformal factor on $M_{u}-\left\{\ell_{u}\right\}$ for which the metric becomes scalar-flat Kähler and asymptotically flat. While the latter follows immediately from the argument of [19], we prefer to give a more elegant argument here that sheds more light on the situation. We begin by giving a new proof of the following theorem of Pontecorvo:

Proposition 5. [26]. Let $\wp: Z \rightarrow M$ be the twistor fibration of a (not necessarily compact) half-conformally-flat conformal Riemannian 4-manifold $(M,[g])$. Let $D \subset Z$ be a complex hypersurface that is the image of a section of $\wp$, and let $J$ denote the complex structure on $M$ corresponding to this section. Let $\bar{D}$ denote the image of $D$ under the real structure $\sigma: Z \rightarrow Z$. Then there exists a metric $g$ in the conformal class $[g]$ such that $(M, g, J)$ is Kähler iff the divisor line bundle of $-2[D]-2[\bar{D}]$ is isomorphic to the canonical line bundle $\kappa$ of $Z$.

Proof. Let us first remark [13] that the vertical tangent bundle of $\wp$ is a smooth complex line bundle whose first Chern class is $\frac{1}{2} c_{1}(Z)$, so that there is a unique holomorphic square root $\kappa^{-1 / 2}$ of $\kappa^{*}$ whose underlying smooth line bundle is isomorphic to the vertical tangent bundle. To say that the divisor line bundle of $-2[D]-2[\bar{D}]$ is isomorphic to the canonical line bundle $\kappa$ is then equivalent to saying that $\kappa^{-1 / 2}$ admits a nontrivial holomorphic section that vanishes at $D$ and $\bar{D}$. Let us orient $M$ so that it is anti-self-dual. By the Penrose transform [12,3], a holomorphic section of $\kappa^{-1 / 2}$ then corresponds to a section of $\odot^{2} \mathbb{S}_{+}=\Lambda_{+}[-3]$ that is in the kernel of the twistor operator

$$
\begin{aligned}
\mathrm{T}: \Gamma\left(M, C^{\infty}\left(\bigodot^{2} \mathbb{S}_{+}\right)\right) & \rightarrow \Gamma\left(M, C^{\infty}\left(\mathbb{S}_{-} \otimes \bigodot^{3} \mathbb{S}_{+}\right)[-2]\right) \\
\omega^{A B} & \mapsto \nabla_{A^{\prime}}^{(A} \omega^{B C)} ;
\end{aligned}
$$

here $\mathbb{S}_{ \pm}$are the spin bundles of $(M,[g])$, while [-3] and [-2] are examples of conformal weight line bundles, defined in such a way that [-4] is the real line bundle $\wedge^{4} T^{*} M$, which is trivialized by the volume form of any metric we choose in the conformal class $[g]$. Since $\sigma$ induces a complex-conjugation on 
$\Gamma\left(Z, \mathscr{O}\left(\kappa^{-1 / 2}\right)\right)$, we may, by taking real and imaginary parts, assume that the section $s$ is $\sigma$-invariant, which corresponds to taking twisted self-dual 2-form $\omega$ to be real. The requirement that $s$ vanish at $D$ and $\bar{D}$ precisely means that $\omega$ must be of type $(1,1)$ with respect to $J$.

Now, if there exists a Kähler metric $g$ in the conformal class, we may just define $\omega$ to be its Kähler form, interpreted as having the appropriate conformal weight. Since the Kähler form is automatically parallel with respect to the Levi-Civita connection of $g$, it is certainly in the kernel of the conformally invariant operator $T$, showing that the divisor corresponding to $\pm J$ is halfanti-canonical, as claimed.

If, conversely, $D \amalg \bar{D}$ is the zero locus of a $\sigma$-invariant section $s$ of $\kappa^{-1 / 2}$, we may construct a family of cohomology classes $\left[s^{-m}\right] \in H^{1}\left(Z, \kappa^{m / 2}\right), m>$ 0 , by covering $Z$ with the 2-patch cover $\{Z-D, Z-\bar{D}\}$, and taking the Čech class of $s^{-m} \in \Gamma\left((Z-D) \cap(Z-\bar{D}), \mathscr{O}\left(\kappa^{m / 2}\right)\right)$. (More abstractly, this amounts to considering the image of $(i / 2,-i / 2)$ under the connecting homomorphism

$$
H^{0}(D, \mathscr{O}) \oplus H^{0}(\bar{D}, \mathscr{O}) \stackrel{\delta}{\rightarrow} H^{1}\left(Z, \mathscr{O}\left(\kappa^{m / 2}\right)\right),
$$

induced by the short exact sequence

$$
\left.0 \rightarrow \mathscr{O}\left(\kappa^{m / 2}\right) \stackrel{s^{\otimes m}}{\rightarrow} \mathscr{O} \rightarrow \mathscr{O}\left|\mathscr{I}_{D}^{m} \oplus \mathscr{O}\right| \mathscr{I}_{\bar{D}}^{m} \rightarrow 0 .\right)
$$

The class $\left[s^{-2}\right] \in H^{1}(Z, \mathscr{O}(\kappa))$ has the Penrose transform interpretation of being a solution $\widetilde{\omega} \in \Gamma\left(M, C^{\infty}\left(\wedge_{+}\right)\right)$of $d \widetilde{\omega}=0$; by using the Penrose transform in the form of the original explicit integral formulae of Penrose [24], one observes, moreover, that this self-dual 2 -form is of type $(1,1)$ with respect to $J$. This is then the Kähler form of a scalar-flat Kähler metric in the conformal class. In fact, this metric is given to us in a quite explicit form, for the class $s^{-1} \in H^{1}\left(Z, \mathscr{O}\left(\kappa^{1 / 2}\right)\right)$ may be interpreted, via the Penrose transform, as a solution $\phi \in \Gamma\left(M, C^{\infty}[-1]\right)$ of the conformal Laplace equation

$$
(\triangle+R / 6) \phi=0
$$

that is real and nonzero on $M$; viewing $\phi$ as a conformal factor then amounts to choosing a metric $g$ in the conformal class so that $\phi \equiv 1$, thus forcing the scalar curvature $R$ of $g$ to vanish. The statement that $s \cdot\left[s^{-2}\right]=\left[s^{-1}\right]$ then becomes the statement that, with respect to this conformal scale, $\widetilde{\omega}=\omega$, so that $\nabla \omega=T \omega+d \omega=0$, and the metric $g$ is Kähler, with Kähler form $\omega$.

Combining this with some key observations due to Poon [28], we obtain

Proposition 6. Let $Z$ be a compact twistor space with a degree 1 divisor $D$ that is not disjoint from its conjugate divisor $\bar{D}$. Then $D \cap \bar{D}$ consists of exactly one real twistor line $L$ corresponding to a point $\ell$ of the 4-manifold $M$. The antiself-dual conformal metric on $M$ given by the twistor construction is represented on $\Sigma:=M-\{\ell\}$ by an asymptotically-flat scalar-flat Kähler metric. Moreover, the underlying complex surface of $\Sigma$ is $\mathbb{C}^{2}$ blown up at a finite number of points. 
Proof. Let $z$ be a point of $D \cap \bar{D}$. Then the $\sigma$-invariance of $D \cap \bar{D}$ implies that $\sigma(z)$ is also in $D \cap \bar{D}$. The real twistor line $L:=\wp^{-1}(\wp(z))$ thus meets $D$ in at least 2 points. Since $[D] \cdot[L]=1$, we must have $L \subset D$, and hence $L \subset D \cap \bar{D}$. But the normal bundle of $D$ restricted to $L$ must now be of degree 1 , while the twistor line $L$ has normal bundle $\mathscr{O}(1) \oplus \mathscr{O}(1)$ in $Z$. Hence the self-intersection of $L$ in $D$ must be 1 .

By surface classification, $D$ is a blowup of $\mathbb{C P}_{2}$, and $L \subset D$ is a projective line avoiding the exceptional set. We now observe that $D \cap \bar{D}$ must therefore consist precisely of $L$, since any other point of the intersection would necessarily be on a projective line $L^{\prime}$ disjoint from $L$. Thus the projection $\left.\wp\right|_{D}: D \rightarrow M$ collapses $L$ to a point $\ell$, but is otherwise a diffeomorphism. Hence $M \approx \overline{\mathbb{C P}}_{2} \# \cdots \# \overline{\mathbb{C P}}_{2}$, and in particular, $M$ is simply connected. Since, by the Ward correspondence $[29,2,12], H^{1}(Z, \mathscr{O}) / H^{1}(Z, \mathbb{Z})$ is the set of anti-self-dual connections mod gauge on the trivial complex line bundle over $M$, and since, $M$ being compact, Hodge theory implies that all such connections are flat, it follows that $H^{1}(Z, \mathscr{O})=0$. Thus holomorphic line bundles on $Z$ are classified by their Chern classes-indeed, by their Chern classes with real coefficients, since $Z$, like $M$, must be simply connected. Since $[D]+[\bar{D}]$ and $\kappa^{-1 / 2}$ have real Chern classes in the $(-1)$-eigenspace of $\sigma$, which is 1 dimensional by the Leray-Hirsch theorem, and since both have degee 2 on a twistor line, we have $\kappa^{-1 / 2} \cong[D]+[\bar{D}]$ as a holomorphic line bundle. Notice that this isomorphism of course persists when we restrict our attention to the twistor space $Z-L$ of $M-\{\ell\}$.

By Proposition 5, the twistorially-induced conformal metric, therefore, is represented on $M-\{\ell\}$ by a scalar-flat Kähler metric $g$. We now need merely show that this metric is asymptotically flat. For this it suffices to observe that $\left[s^{-1}\right] \in H^{1}\left(Z-L, \kappa^{1 / 2}\right)$ is the restriction to $Z-L$ of the extension class of the exact sequence

$$
\begin{aligned}
& 0 \rightarrow \mathscr{J}_{D \cup \bar{D}} \quad \rightarrow \quad \mathscr{I}_{D} \oplus \mathscr{F}_{\bar{D}} \quad \rightarrow \quad \mathscr{I}_{L} \rightarrow 0 \\
& f \quad \mapsto\left(f \otimes s_{2},-f \otimes s_{1}\right) \\
& (g, h) \quad \mapsto g \otimes s_{1}+h \otimes s_{2}
\end{aligned}
$$

where $s_{1}$ and $s_{2}$ represent the canonical sections of $[D]$ and $[\bar{D}]$, respectively, so normalized so that $s=\frac{1}{2} s_{1} \otimes s_{2}$; here $\mathscr{I}_{X} \subset \mathscr{O}_{Z}$ denotes the ideal sheaf of a subvariety $X \subset Z$. But this extension class $\in \operatorname{Ext}_{Z}^{1}\left(\mathscr{F}_{L}, \kappa^{1 / 2}\right)$ is precisely the Serre class of the line $L$, and the corresponding field $\phi$, therefore, is [1] the conformal Green's function of $(M, \ell)$, with a singularity $1 / r^{2}$ at $\ell$.

We these tools in hand, we can now prove our main result:

Theorem 3. Let $\mathbb{C}^{2}$ be blown up at $n$ points, and let a Kähler class be specified on the blowup. If the given points are nearly collinear, there is an asymptotically flat scalar-flat Kähler metric in the given Kähler class. 
Proof. The result we will prove may be more precisely stated as follows: In the space of configurations of $n$ positively weighted points in $\mathbb{C}^{2}$, there is a neighborhood of the weighted collinear configurations for which each corresponding blow up admits an asymptotically flat scalar-flat Kähler metric for which the areas of the exceptional divisors are the given weights. Moreover, the metric we produce will be uniquely determined by the assumption that it be sufficiently near one of the model metrics [20] whose twistor spaces are described in $\S 2$.

To do this, we apply Proposition 6 to the real versal family of Theorem 2, which is a real slice of the versal family of Proposition 1 . Since the latter induces all small deformations of $(D, L) \amalg(\bar{D}, L)$ by Proposition 4 , the real versal family of $(Z, \sigma, D)$ induces all small deformations of $\left(D, L,\left.\sigma\right|_{L}\right)$. The latter correspond to configurations of blow-up points in $\mathbb{C}^{2}$ modulo similarity transformations. Corresponding to a choice of marking and a base point for $L$ as in the discussion leading to Proposition 2 is a choice of 2 of the blow-up points in $\mathbb{C}^{2}$ and a real 3-dimensional half space with its edge on the line through them. By a suitable similarity transformation, we may take these points to be the points $(0,0)$ and $(1,0)$ of $\mathbb{C}^{2}$, and take the 3-dimensional half space to be $\Re z_{2}=0, \Im z_{2} \geq 0$. An open neighborhood of a given collinear configuration of distinct points in the space of these "marked" configurations may then be identified with an open set in $\left(\mathbb{C}^{2}\right)^{n-2}$, and the corresponding space of weighted marked configurations is an open set in $\left(\mathbb{C}^{2}\right)^{n-2} \times\left(\mathbb{R}^{+}\right)^{n} \approx \mathbb{R}^{5 n-8}$. There is thus a natural smooth map from the (5n-8)-dimensional parameter space $U$ of our real deformation to $\left(\mathbb{C}^{2}\right)^{n-2} \times\left(\mathbb{R}^{+}\right)^{n}$ that reads off the configuration corresponding to $\left(D, L, p,\left.\sigma\right|_{L}\right)$ with respect to the given marking and the $n$-tuple of areas of the exceptional divisors. ${ }^{5}$ Since [20] this map is a diffeomorphism when restricted to the family of weighted collinear configurations, and since the composition of this map with projection to the $(4 n-8)$-dimensional space of unweighted configurations has maximal rank by Proposition 4, the derivative of this map is an isomorphism at the base point $o \in U$ representing an arbitrary collinear configuration of $n$ distinct points. The claim now follows immediately from the inverse function theorem.

Remark. For this proof, it was not necessary to understand the manner in which the Kähler class is encoded by the twistor space. It is nonetheless worth pointing out that this information can be read off in a fairly simple manner. Indeed, let $Z$ be the twistor space of any scalar-flat Kähler surface and let $D$ be the divisor corresponding to the complex structure. Since the Kähler form gives a section of $\kappa^{-1 / 2}$ vanishing along $D$ in a nondegenerate manner, ${ }^{6}$ the normal bundle $\nu_{D}$ of $D \subset Z$ is $\left.\kappa^{-1 / 2}\right|_{D}$. Thus $\left.\kappa^{-1}\right|_{D}=\nu_{D} \otimes \Lambda^{2} T D=\left.\kappa^{-1 / 2}\right|_{D} \otimes \Lambda^{2} T D$, and hence $\nu_{D}=\left.\kappa^{-1 / 2}\right|_{D}=\bigwedge^{2} T D$. The tangent bundle of $Z$, therefore, may be

\footnotetext{
${ }^{5}$ Recall that the base point $p$ is not an arbitrary point of $L$, but rather is required to lie on the equatorial $S^{1}$ determined by $\left.\sigma\right|_{L}$ and the point $0_{u}$ determined by the homology marking of $D$.

${ }^{6}$ Of course this argument, therefore, only applies in our examples away from the line $L$ !
} 
viewed as an extension

$$
\left.0 \rightarrow T D \rightarrow T Z\right|_{D} \rightarrow \bigwedge^{2} T D \rightarrow 0
$$

and so has an extension class in $H^{1}\left(D, \mathscr{O}\left(T D \otimes \bigwedge^{2} T^{*} D\right)\right)=H^{1}\left(D, \Omega^{1}\right)$. Using the definition (cf. $\S 1$ ) of the complex-structure tensor $J$ of $Z=S\left(\bigwedge_{+}\right)=$ $S\left(\mathbb{R} \oplus \Omega^{2}\right)$, one may verify by direct computation that this class is given by the Dolbeault class of the Kähler form.

\section{ACKNOWLEDGMENTS}

The author would like to thank the Forschungsinstitut für Mathematik of the ETH Zürich for its hospitality during the inception of the present work. In addition, he would like to express his appreciation to Simon Donaldson, Robert Friedman, Yat-Sun Poon, and Massimiliano Pontecorvo for their useful suggestions and comments.

\section{REFERENCES}

1. M. Atiyah, Green's functions for self-dual four-manifolds, Adv. Math. Suppl. Stud., vol. 7 Academic Press, New York, 1981, pp. 129-158.

2. M. Atiyah, N. Hitchin, and I. Singer, Self-duality in four dimensional Riemannian geometry, Proc. Roy. Soc. London Ser. A 362 (1978), 425-461.

3. R. Baston and M. Eastwood, The Penrose transform: Its interaction with representation theory, Oxford Univ. Press, London-New York, 1990.

4. E. Calabi, Extremal Kähler metrics, Seminar on Differential Geometry (S. T. Yau, ed.), Ann. of Math. Stud., vol. 102 Princeton Univ. Press, Princeton, NJ, 1982, pp. 259-290.

5. S. K. Donaldson and R. Friedman, Connected sums of self-dual manifolds and deformations of singular spaces, Nonlinearity 2 (1989), 197-239.

6. E. J. Flaherty, The non-linear graviton in interaction with a photon, Gen. Rel. Grav. 9 (1978), 961-978.

7. A. Floer, Self-dual conformal structures on $\ell \mathbb{C P} \mathbb{P}^{2}$, J. Differential Geometry 33 (1991), 551573.

8. A. Fujiki, On a compact complex manifold in $\mathscr{C}$ without holomorphic 2-forms, Publ. RIMS 19 (1983), 193-202.

9. P. Gauduchon, Surfaces Kählériennes dont la courbure vérifie certaines conditions de positivité, Géometrie Riemannienne en Dimension 4, Séminaire A. Besse, 1978/1979, (Bérard-Bergery, Berger, and Houzel, eds.), CEDIC/Fernand Nathan, 1981.

10. H. Grauert and R. Remmert, Coherent analytic sheaves, Springer-Verlag, Berlin-HeidelbergNew York, 1984.

11. N. J. Hitchin, Polygons and gravitons, Math. Proc. Cambridge Philos. Soc. 83 (1979), 465476.

12. L Linear field equations on self-dual spaces, Proc. Roy. Soc. London. Ser. A 370 (1980), 173-191.

13. __ Kählerian twistor spaces, Proc. London. Math. Soc. (3) 43 (1981), 133-150.

14. __ Complex manifolds and Einstein's equations, Lecture Notes in Math., vol. 970, 1982, pp. 73-99.

15. P. E. Jones and K. P. Tod, Minitwistor spaces and Einstein-Weyl spaces, Class. Quantum Grav. 2 (1985), 565-577. 
16. K. Kodaira, A theorem of completeness of characteristic systems for analytic families of compact submanifolds of complex manifolds, Ann. of Math. 75 (1962), 146-162.

17. K. Kodaira, L. Nirenberg, and D. Spencer, On the existence of deformations of complex analytic structure, Ann. of Math. 68 (1958), 450-459.

18. K. Kodaira and D. Spencer, On deformations of complex analytic structure, I, II, III, Ann. of Math. (2) 67 (1958), 328-466; 71 (1960), 43-76.

19. C. LeBrun, Poon's self-dual metrics and Kähler geometry, J. Differential Geom. 28 (1988), 341-343.

20. $ـ$, Explicit self-dual metrics on $\mathbb{C P}_{2} \# \cdots \# \mathbb{C P}_{2}$, J. Differential Geom. 34 (1991), 223253.

21. _ Scalar-flat Kähler metrics on blown-up ruled surfaces, J. Reine Angew. Math. $\mathbf{4 2 0}$ (1991), 161-177.

22. Self-dual manifolds and hyperbolic geometry, Proceedings of the 1990 Taneguchi Symposium, (T. Mabuchi, ed.), Lecture Notes in Math., Springer-Verlag, New York (to appear).

23. C. LeBrun and Y. S. Poon, Twistors, Kähler manifolds, and bimeromorphic geometry. II, J. Amer. Math. Soc. 5 (1992), 317-325 (this issue).

24. R. Penrose, Solutions of the zero-rest-mass equations, J. Math. Phys. 10 (1969), 61-99.

25. Non-linear gravitons and curved twistor theory, Gen. Rel. Grav. 7 (1976), 31-52.

26. M. Pontecorvo, On twistor spaces of anti-self-dual Hermitian surfaces, Trans. Amer. Math. Soc. (to appear).

27. Y. S. Poon, Compact self-dual manifolds with positive scalar curvature, J. Differential Geom. 24 (1986), 97-132.

28. _ Algebraic structure of twistor spaces, preprint, 1990.

29. R. Ward, On self-dual gauge-fields, Phys. Lett. A 61 (1977), 81-82.

30. S.-T. Yau, Problem section, Seminar on Differential Geometry, Ann. of Math. Stud., vol. 102, Princeton Univ. Press, Princeton, NJ, 1982.

ABsTRACT. By considering deformations of the Moishezon twistor spaces of $\mathbb{C P}_{2} \# \ldots \# \mathbb{C P}_{2}$ constructed in [20], we show that the blow up of $\mathbb{C}^{2}$ at $n$ points in general position admits an asymptotically flat scalar-flat Kähler metric in each Kähler class, at least provided that the given points are nearly collinear.

Department of Mathematics, SUNY at Stony Brook, Stony Brook, New York, 11794

E-mail address: claude@math.sunysb.edu 